\title{
27. ALKENONE SEA-SURFACE TEMPERATURES AND CARBON BURIAL AT SITE 846 (EASTERN EQUATORIAL PACIFIC OCEAN): THE LAST 1.3 M.Y.
}

\author{
K.-C. Emeis, ${ }^{2}$ H. Doose, ${ }^{2}$ A. Mix,${ }^{3}$ and D. Schulz-Bull ${ }^{4}$
}

\begin{abstract}
We analyzed the unsaturation ratio $\left(\mathrm{U}_{37}^{\mathrm{k}}\right)$ of long-chain ketones- a molecular sea-surface temperature (SST) indicator-concentrations of carbonate and organic carbon in sediments from Site 846 in the eastern equatorial Pacific Ocean. Based on an isotopic age model for the composite depth section of $0-46 \mathrm{~m}$ below seafloor and on estimates of sediment density, accumulation rates of these biogenic compounds were calculated. Our combined temperature and biogenic flux record traces conditions at the origin of the South Equatorial Current over the last 1.3 m.y.

SSTs have fluctuated considerably over the interval studied. Along trend of gradual decrease from $24^{\circ} \mathrm{C}$ at $1.3 \mathrm{Ma}$ ends between 500 and $400 \mathrm{Ka}$, when lowest values of $19^{\circ} \mathrm{C}$ were reached. Since this time, the temperature data indicate a warming trend to the Holocene modulated by high-amplitude variation $\left(19^{\circ}\right.$ to $\left.27^{\circ} \mathrm{C}\right)$. The inversion of the trend between 400 and $500 \mathrm{Ka}$ coincides with maximal accumulation rates of carbonate, which since then have decreased. In contrast, organic carbon accumulation since then has increased in variability and in absolute values.

On shorter time scales, the records show a strong link to the global climatic background. Since $1.3 \mathrm{Ma}$, carbonate $\left(0.2-3 \mathrm{~g} / \mathrm{cm}^{2}\right.$ k.y. $\left.{ }^{-1}\right)$ and organic carbon accumulation rates $\left(2-30 \mathrm{mg} / \mathrm{cm}^{2} \mathrm{k} . \mathrm{y}^{-1}\right)$ were consistently high (more than twice their modern values and those of interglacials) during glacial maxima in the benthic isotope record, when temperatures were low.

However, cross-spectral analyses with the $\delta^{18} \mathrm{O}$ record suggest that variation in organic carbon flux is not linked directly to variations in SST. Temperature maxima in our record led interglacial events by 7 k.y. in the 100-k.y. eccentricity cycle and by 5 k.y. in the 41-k.y. obliquity cycle. In contrast, maxima in organic carbon accumulation lag behind glacial maxima and low temperatures by 14 k.y. in the eccentricity cycle. On glacial/interglacial time scales, a prominent influence on SST - but not on organic carbon burial—at Site 846 appears to be the advection of cold water into the South Equatorial Current.
\end{abstract}

\section{INTRODUCTION}

The primary objective of Ocean Drilling Program (ODP) Leg 138 was to reconstruct the paleoceanographic history of the eastern equatorial Pacific Ocean, an area that is characterized today by intense gradients in physical, chemical, and biological properties of surface waters. Among the diagnostic characteristics of each water mass interacting here are temperatures at the sea surface and biological productivity in the euphotic zone (e.g., Wooster, 1969; Bryden and Brady, 1985; Chavez et al., 1990; Chavez and Barber, 1987).

Site 846 of Leg $138\left(3^{\circ} 05.696^{\prime} \mathrm{S}\right.$ and $90^{\circ} 49.078^{\prime} \mathrm{W}$ in a water depth of $3307 \mathrm{~m}$ ) is situated $300 \mathrm{~km}$ south of Galapagos (Fig. 1). It is located in the southeastern origin of the cold tongue of the Equatorial Pacific Ocean (Wyrtki, 1981), where the Peru Current merges with the South Equatorial Current (SEC). Here, combined eastward shoaling of the Equatorial Undercurrent (EUC) and advection of water from the eastern boundary current result in high nutrient levels in the euphotic zone, high biological production, and cold SSTs (Chavez and Barber, 1987; Halpern et al., 1990).

The extent of the cold tongue, which is defined by meridional temperature gradients of $0.5^{\circ} \mathrm{C}$ per $100 \mathrm{~km}$ north and south of the equator, is traced by the unsaturation ratio of long-chain ketones in surface sediments (Fluegge, unpubl. data), which are a robust and accurate molecular thermometer (Prahl et al., 1988; Eglinton et al., 1992; Lyle et al., 1992). Today, temperatures at the site display a pronounced annual cycle that ranges from $20^{\circ}$ to $27^{\circ} \mathrm{C}$ (Halpern et al., 1990). SSTs of the climatological mean in the period from July to September range from $21^{\circ}$ to $22^{\circ} \mathrm{C}$; modern observations indicate

\footnotetext{
'Pisias, N.G., Mayer, L.A., Janecek, T.R., Palmer-Julson, A., and van Andel, T.H (Eds.), 1995. Proc. ODP, Sci. Results, 138: College Station, TX (Ocean Drilling Program).

${ }^{2}$ Geologisch-Paläontologisches Institut, Universität Kiel, Federal Republic of Germany.

${ }^{3}$ School of Oceanography, Oregon State University, Corvallis, OR, U.S.A.

${ }^{4}$ Institut für Meereskunde, Universität Kiel, Federal Republic of Germany.
}

significant interannual variability $\left(2^{\circ} \mathrm{C}\right)$. During the main period of annual plankton productivity in early austral winter (Dandonneau and Eldin, 1987), temperatures at the site average $21^{\circ}$ to $23^{\circ} \mathrm{C}$ and coincide with intensified southeast trade winds and maximal intensity of the Peru Current.

According to surveys by Chavez et al. (1990), dominant primary producing organisms in the eastern equatorial Pacific are blue green algae, picoplankton, dinoflagellates, and diatoms. Coccolithophores, which produce the alkenones used for temperature reconstructions, are among the dominant phytoplankton taxa, but do not contribute significantly to primary productivity in the eastern equatorial Pacific Ocean. However, their abundance correlates well with measured chlorophyll concentrations in surface waters during peak primary production (Chavez et al., 1990), and we expect that the alkenone unsaturation index records temperatures at the time of highest annual productivity. This assumption is corroborated by a survey of the $U_{37}^{k}$ indexes in core-top samples: Fluegge (unpubl. data) found an alkenone temperature of $22.5^{\circ} \mathrm{C}$ close to the location of Site 846 . His contour maps of modern alkenone SSTs in the area depict a strong temperature gradient over a range of $22^{\circ}$ to $24^{\circ} \mathrm{C}$, and the zone of low temperatures corresponds well with the area of highest TOC accumulation rates in coretop samples (Lyle, 1992).

Site 846 was located to explore the history of interaction between the eastern boundary current and the SEC, both of which are strongly influenced by changes in the atmospheric circulation of the Southern Hemisphere on seasonal, interannual, and geological time scales. As a first contribution to this end, we analyzed concentrations and accumulation rates of organic carbon and carbonate, and the unsaturation ratio of long-chain alkenones in lipid extracts of samples from the top $46 \mathrm{~m}$ of the sedimentary record recovered at Site 846.

\section{METHODS}

Drilling four offset holes recovered a continuous and highly resolved sediment record at Site 846 (Mayer, Pisias, Janecek, et al., 


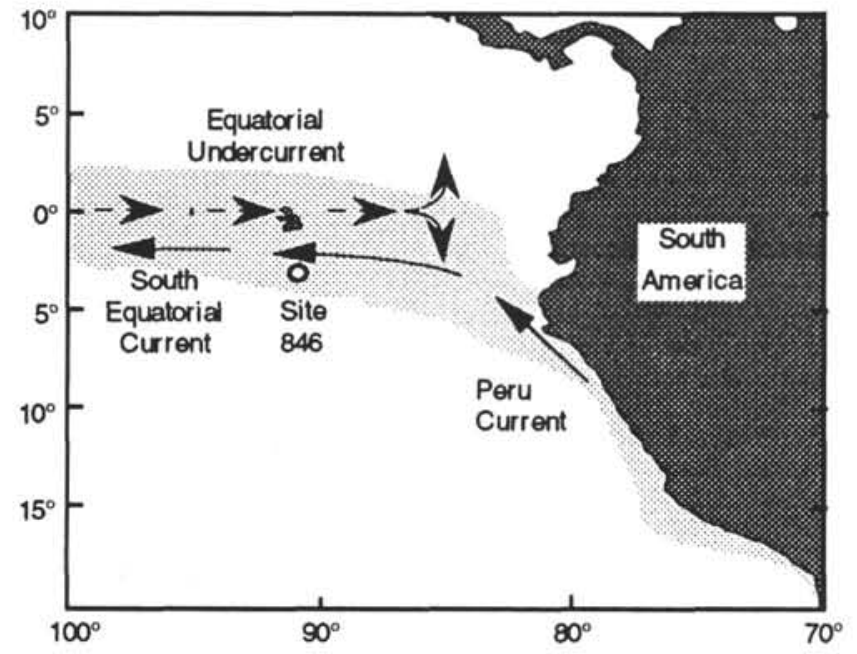

Figure 1. Location of Site 846 in the eastern Pacific Ocean. Major currents affecting the surface hydrography at Site 846 are indicated. The cold tongue extends off South America and is marked by low SST and high nutrient concentrations (after Wooster, 1969).

1992). Age assignments for our samples are based on their position in the revised composite depth section (revised meters composite depth [rmcd]; Hagelberg et al., this volume) and the corresponding depth/age model of Mix et al. (this volume). Average sample interval of our accumulation rate time series is 5.5 k.y. (maximum of 24 k.y.), that of SST is 6.5 k.y. (maximum of 24 k.y.). The mean sampling interval of the isotope record is 2.6 k.y. (maximum of 21 k.y.). To compute accumulation rates of individual components from concentration data, we adopted linear sedimentation rates from Mix et al. (this volume) and interpolated for each sample interval of our sample set.

Comparing the smoothed GRAPE wet-bulk density (WBD) record of the composite section with dry-bulk density (DBD) measurements on discrete samples (Mayer, Pisias, Janecek, et al., 1992), we estimated DBD of each sample in our set based on the corresponding GRAPE density value in the composite depth scale (Fig. 2) as

$$
\operatorname{DBD}\left(\mathrm{g} / \mathrm{cm}^{3}\right)=-1.48+1.4 \times \text { GRAPE density. }
$$

Mass accumulation rates (MAR) and accumulation rates of organic carbon (TOC MAR) and carbonate $\left(\mathrm{CaCO}_{3} \mathrm{MAR}\right)$ were calculated as the product of concentration, linear sedimentation rate, and DBD estimates as follows:

$$
\begin{gathered}
\operatorname{MAR}\left(\mathrm{g} / \mathrm{cm}^{2} \mathrm{k} \cdot \mathrm{y} \cdot{ }^{-1}\right)=\operatorname{LSR}(\mathrm{cm} / \mathrm{k} \cdot \mathrm{y} .) \times \mathrm{DBD}\left(\mathrm{g} / \mathrm{cm}^{3}\right), \\
\operatorname{TOC} \operatorname{MAR}\left(\mathrm{mg} / \mathrm{cm}^{2} \mathrm{k} \cdot \mathrm{y} \cdot{ }^{-1}\right)=(\operatorname{MAR} / 100 \times \% \mathrm{TOC}) \times 1000, \\
\mathrm{CaCO}_{3} \operatorname{MAR}\left(\mathrm{g} / \mathrm{cm}^{2} \mathrm{k} \cdot \mathrm{y} \cdot{ }^{-1}\right)=\operatorname{MAR} / 100 \times \% \mathrm{CaCO}_{3} .
\end{gathered}
$$

Total carbon (TC) concentrations were measured as $\mathrm{CO}_{2}$ in a Carlo Erba Model 1108 elemental analyzer after flash combustion of sample in a tin foil container at approximately $1600^{\circ} \mathrm{C}$ (oxidation column temperature $1020^{\circ} \mathrm{C}$ ) in helium carrier gas, catalytic oxidation, chromatographic separation, and detection in a thermal conductivity detector. Calculation of response factors of standards (acetanilide; one standard and one blank run for each 10 sample runs) and corrections for blanks permitted us to calculate weight percentages of carbon and nitrogen in the sample. Duplicate analyses of samples indicate that the standard deviation of total carbon analyses is less than $0.1 \%(n=62)$. To measure organic carbon, we acidified a weighed split of sample (approximately $10 \mathrm{mg}$ ) in a tin foil container with successively stronger phosphoric acid (maximum 17\%) until no further $\mathrm{CO}_{2}$ evolved. The acidified sample containers were then dried at $40^{\circ} \mathrm{C}$ in a desiccator and analyzed before the tin foil containers disintegrated. All samples were

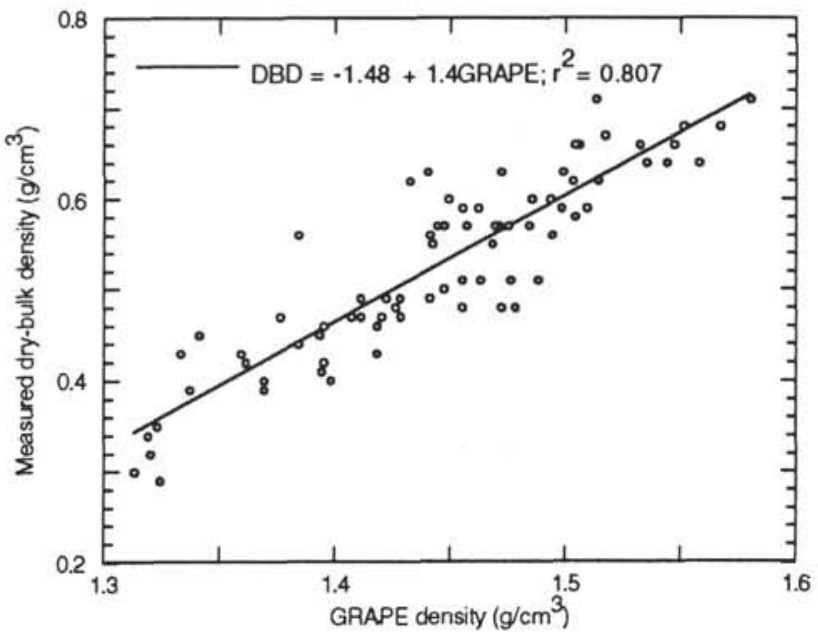

Figure 2. Correlation between measured DBD of discrete samples and stacked GRAPE density of corresponding intervals (Mayer, Pisias, Janecek, et al., 1992).

run in duplicate. Standard deviations average $0.05 \%$ TOC. Concentration of $\mathrm{CaCO}_{3}$ content was calculated by assuming that the difference between total and organic carbon is carbonate carbon:

$$
\% \mathrm{CaCO}_{3}=(\mathrm{TC}-\mathrm{TOC}) \times 8.3334 .
$$

To reconstruct SSTs, we measured the unsaturation ratio of longchain ketones produced by coccolithophores of the family Gephyrocapsaceae in lipid extracts of sediments (Brassell et al., 1986). Weighed splits of dried and homogenized sediments $(0.3-3 \mathrm{~g})$ were extracted twice in an ultrasonic bath for 40 min each. The solvent was $2 \times 20 \mathrm{~mL}$ twice-distilled $\mathrm{CH}_{2} \mathrm{Cl}_{2}$, spiked with a known amount of $5 \alpha$-cholestane as an internal recovery standard. After each extraction step, samples were centrifuged and the solvent was collected by pipette. The two lipid extracts were combined and lipid classes were separated chromatographically on commercial silica columns (conditioned with $5 \mathrm{~mL}$

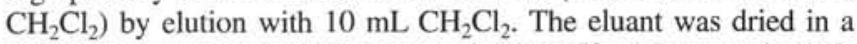
rotary evaporator and redissolved in 10,20 , or $50 \mu \mathrm{L}$ hexane, depending on original sediment weight and TOC concentration. The sample in hexane $(1 \mu \mathrm{L})$ was injected in split mode onto a Fisons Instruments gas chromatograph fitted with a 50-m glass capillary column (Ultra-1; i.d., $0.35 \mathrm{~mm}$, film thickness, $0.52 \mathrm{~mm}$ ) and a flame ionization detector. Hydrogen was used as carrier gas (column head pressure $140 \mathrm{kPa}$ ). The temperature program was $150^{\circ}$ to $300^{\circ} \mathrm{C}$ at $10^{\circ} / \mathrm{min}, 300^{\circ}$ to $330^{\circ} \mathrm{C}$ at $2^{\circ} \mathrm{C} / \mathrm{min}$, and isothermal at $330^{\circ} \mathrm{C}$ for $18 \mathrm{~min}$.

Alkenones were identified by comparing retention times with those of synthetic standards (provided by G. Eglinton, Bristol). Peak areas were converted to the $\mathrm{U}_{37}^{\mathrm{k}}$ index, and SSTs calculated according to a calibration based on culture experiments (Prahl et al., 1988). For the sake of clarity and compatibility with other temperature proxy indicators, we report our results based on the $\mathrm{U}_{37}^{\mathrm{k}}$ index as temperatures (Prahl et al., 1988):

$$
\operatorname{SST}\left({ }^{\circ} \mathrm{C}\right)=\left(\mathrm{C}_{37: 2} /\left(\mathrm{C}_{37: 2}+\mathrm{C}_{37: 3}\right)-0.039\right) / 0.034 \text {. }
$$

Results of duplicate analyses $(n=16)$ indicate that the standard deviation of temperatures calculated from $\mathrm{U}_{37}^{\mathrm{k}}$ is $0.15^{\circ} \mathrm{C}$ (range $\left.0-0.35^{\circ} \mathrm{C}\right) . \mathrm{s}$

\section{RESULTS}

Table 1 is a list of samples and analytical results from Site 846. Samples are identified based on standard ODP sample identification (hole, core, section, interval), shipboard depth in meters below seafloor, and a composite depth assigned to each sample based on the spliced records for all four holes drilled at Site 846. Figure 3 shows 
Table 1. Analytical results of chemical analyses for samples of Site $\mathbf{8 4 6}$.

\begin{tabular}{|c|c|c|c|c|c|c|c|c|c|c|c|}
\hline $\begin{array}{l}\text { Core, section, } \\
\text { interval }(\mathrm{cm})\end{array}$ & $\begin{array}{l}\text { Depth } \\
\text { (mbsf) }\end{array}$ & $\begin{array}{l}\text { Rev } \\
\text { med }\end{array}$ & Age & LSR & WBD & DBD & BAR & $\begin{array}{c}\text { Mean } \\
\text { TC }\end{array}$ & $\mathrm{CaCO}_{3}$ & $\mathrm{CaCO}_{3}$-AR & $\begin{array}{l}\text { Mean } \\
\text { TOC }\end{array}$ \\
\hline 138-846- & & & & & & & & & & & \\
\hline A1-1, 5-7 & 0.06 & 0.07 & 2.2 & 5.9 & 1.36 & 0.41 & 2.4 & 8.9 & 66.0 & 1.6 & 1.03 \\
\hline A1- $1,20-22$ & 0.21 & 0.25 & 6.1 & 4.3 & 1.41 & 0.48 & 2.0 & 9.7 & 75.3 & 1.5 & 0.62 \\
\hline A $1-1,40-42$ & 0.41 & 0.56 & 13.7 & 4.1 & 1.40 & 0.46 & 1.9 & 9.6 & 71.5 & 1.4 & 1.01 \\
\hline A $1-1,60-62$ & 0.61 & 0.73 & 17.7 & 4.3 & 1.38 & 0.43 & 1.8 & 8.9 & 64.3 & 1.2 & 1.15 \\
\hline A $1-1,80-82$ & 0.81 & 0.90 & 21.7 & 4.4 & 1.37 & 0.42 & 1.9 & 9.3 & 66.0 & 1.2 & 1.42 \\
\hline $\mathrm{A} 1-1,100-102$ & 1.01 & 1.05 & 25.0 & 4.6 & 1.37 & 0.43 & 1.9 & 9.4 & 68.2 & 1.3 & 1.22 \\
\hline A $1-1,120-122$ & 1.21 & 1.19 & 28.0 & 4.8 & 1.35 & 0.40 & 1.9 & 8.9 & 62.5 & 1.2 & 1.37 \\
\hline A $1-1,140-142$ & 1.41 & 1.34 & 31.1 & 5.2 & 1.38 & 0.44 & 2.2 & 8.5 & 60.7 & 1.3 & 1.20 \\
\hline A $1-2,20-22$ & 1.71 & 1.69 & 37.7 & 5.5 & 1.37 & 0.42 & 2.4 & 8.0 & 59.7 & 1.4 & 0.79 \\
\hline A $1-2,40-42$ & 1.91 & 1.88 & 40.9 & 6.0 & 1.36 & 0.41 & 2.4 & 7.1 & 52.5 & 1.3 & 0.78 \\
\hline A $1-2,61-63$ & 2.12 & 2.07 & 44.0 & 6.3 & 1.35 & 0.39 & 2.5 & 6.3 & 42.0 & 1.0 & 1.21 \\
\hline Al- $2,80-82$ & 2.31 & 2.23 & 46.5 & 6.5 & 1.33 & 0.37 & 2.4 & 6.0 & 41.9 & 1.0 & 0.99 \\
\hline A $1-2,100-102$ & 2.51 & 2.42 & 49.4 & 6.5 & 1.32 & 0.35 & 2.3 & 6.3 & 46.3 & 1.1 & 0.78 \\
\hline A $1-2,120-122$ & 2.71 & 2.67 & 53.3 & 6.4 & 1.32 & 0.35 & 2.3 & 6.3 & 43.9 & 1.0 & 0.99 \\
\hline Al- $2,140-142$ & 2.91 & 2.89 & 56.8 & 6.0 & 1.33 & 0.37 & 2.2 & 6.2 & 43.9 & 1.0 & 0.91 \\
\hline A1 $-3,12-14$ & 3.13 & 3.15 & 61.3 & 5.5 & 1.32 & 0.35 & 1.9 & 6.0 & 40.6 & 0.8 & 1.09 \\
\hline D1-1, 20-22 & 4.21 & 3.32 & 64.5 & 5.2 & 1.33 & 0.36 & 1.8 & 5.5 & 39.8 & 0.7 & 0.75 \\
\hline A $1-3,35-37$ & 3.36 & 3.35 & 65.2 & 4.7 & 1.31 & 0.33 & 1.6 & 5.7 & 36.1 & 0.6 & 1.38 \\
\hline A $1-3,50-52$ & 3.51 & 3.45 & 67.3 & 4.4 & 1.35 & 0.39 & 1.8 & 5.6 & 35.1 & 0.6 & 1.40 \\
\hline A $1-3,70-72$ & 3.71 & 3.59 & 70.6 & 4.0 & 1.31 & 0.34 & 1.4 & 4.4 & 31.8 & 0.4 & 0.59 \\
\hline D1-1, $60-62$ & 4.61 & 3.76 & 75.1 & 3.7 & 1.26 & 0.27 & 1.0 & 4.5 & 27.5 & 0.3 & 1.14 \\
\hline Al $-3,90-92$ & 3.91 & 3.79 & 76.0 & 3.2 & 1.31 & 0.34 & 1.1 & 5.6 & 40.3 & 0.5 & 0.73 \\
\hline Al-3, $110-112$ & 4.11 & 3.96 & 81.3 & 3.1 & 1.32 & 0.36 & 1.1 & 4.7 & 34.5 & 0.4 & 0.60 \\
\hline Al $-3,130-132$ & 4.31 & 4.10 & 86.1 & 2.8 & 1.30 & 0.32 & 0.9 & 4.7 & 33.0 & 0.3 & 0.79 \\
\hline D $1-1,120-122$ & 5.21 & 4.35 & 95.3 & 2.8 & 1.30 & 0.32 & 0.9 & 4.9 & 31.6 & 0.3 & 0.69 \\
\hline D1-1, 140-142 & 5.41 & 4.58 & 103.4 & 3.0 & 1.31 & 0.34 & 1.0 & 4.7 & 31.2 & 0.3 & 0.93 \\
\hline D1-2, 20-22 & 5.71 & 4.93 & 114.3 & 3.4 & 1.31 & 0.33 & 1.2 & 5.3 & 31.9 & 0.4 & 1.46 \\
\hline D $1-2,40-42$ & 5.91 & 5.15 & 120.2 & 3.9 & 1.34 & 0.39 & 1.5 & 7.6 & 46.0 & 0.7 & 2.04 \\
\hline D1-2, 60-62 & 6.11 & 5.35 & 125.2 & 4.1 & 1.36 & 0.41 & 1.7 & 6.6 & 48.5 & 0.8 & 0.74 \\
\hline D1-2, 80-82 & 6.31 & 5.55 & 129.9 & 4.3 & 1.31 & 0.34 & 1.5 & 7.5 & 48.5 & 0.7 & 0.72 \\
\hline D1-2, $100-102$ & 6.51 & 5.75 & 134.5 & 4.4 & 1.39 & 0.44 & 2.0 & & & & 0.50 \\
\hline D1-2, $120-122$ & 6.71 & 5.95 & 138.9 & 4.6 & 1.42 & 0.49 & 2.2 & 7.8 & 57.2 & 1.3 & 0.98 \\
\hline DI- $2,140-142$ & 6.91 & 6.16 & 143.5 & 4.6 & 1.41 & 0.48 & 2.2 & & & & \\
\hline D $1-3,20-22$ & 7.21 & 6.46 & 149.9 & 4.7 & 1.41 & 0.48 & 2.3 & 6.6 & 49.1 & 1.1 & 0.69 \\
\hline D1-3, $40-42$ & 7,41 & 6.66 & 154.1 & 4.8 & 1.42 & 0.49 & 2.4 & 7.2 & 53.0 & 1.3 & 0.87 \\
\hline D1-3, 60-62 & 7.61 & 6.84 & 157.8 & 4.9 & 1.39 & 0.45 & 2.2 & 7.6 & 56.7 & 1.2 & 0.84 \\
\hline D1-3, 80-82 & 7.81 & 7.03 & 161.7 & 4.8 & 1.37 & 0.42 & 2.0 & 7.0 & 50.4 & 1.0 & 0.98 \\
\hline D1-3, $100-102$ & 8.01 & 7.21 & 165.5 & 4.7 & 1.40 & 0.46 & 2.2 & & & & 1.00 \\
\hline D1 $-3,120-122$ & 8.21 & 7.39 & 169.4 & 4.4 & 1.38 & 0.44 & 2.0 & 7.7 & 57.5 & 1.1 & 0.80 \\
\hline D1-3, 140-142 & 8.43 & 7.58 & 173.9 & 3.8 & 1.37 & 0.42 & 1.6 & 6.5 & 45.7 & 0.8 & 1.05 \\
\hline D $1-4,20-22$ & 8.71 & 7.81 & 180.4 & 3.2 & 1.37 & 0.42 & 1.3 & & & & 1.14 \\
\hline D $1-4,40-42$ & 8.91 & 7.98 & 186.3 & 2.6 & 1.35 & 0.39 & 1.0 & 7.3 & 53.6 & 0.5 & 0.84 \\
\hline D $1-4,60-62$ & 9.11 & 8.13 & 192.6 & 2.2 & 1.37 & 0.43 & 0.9 & 7.5 & 55.6 & 0.5 & 0.83 \\
\hline D1-4, 80-82 & 9.31 & 8.29 & 200.7 & 1.9 & 1.41 & 0.47 & 0.9 & 7.3 & 56.0 & 0.5 & 0.62 \\
\hline Di $-4,100-102$ & 9.51 & 8.44 & 209.1 & 1.8 & 1.40 & 0.47 & 0.8 & & & & 0.42 \\
\hline D $1-4,120-122$ & 9.71 & 8.61 & 218.1 & 2.2 & 1.46 & 0.55 & 1.2 & 7.9 & 60.9 & 0.7 & 0.55 \\
\hline D1 $-4,144-146$ & 9.95 & 8.84 & 227.4 & 3.0 & 1.44 & 0.51 & 1.6 & 8.3 & 62.6 & 1.0 & 0.82 \\
\hline D1-5, 20-22 & 10.21 & 9.11 & 234.8 & 4.1 & 1.45 & 0.53 & 2.3 & & & & 0.73 \\
\hline D1 $-5,40-42$ & 10.41 & 9.32 & 239.0 & 5.4 & 1.41 & 0.48 & 2.7 & 7.4 & 56.7 & 1.5 & 0.61 \\
\hline D1-5, 60-62 & 10.61 & 9.52 & 242.4 & 6.0 & 1.40 & 0.46 & 2.9 & 7.6 & 56.8 & 1.6 & 0.75 \\
\hline D1 $-5,80-82$ & 10.81 & 9.71 & 245.5 & 5.9 & 1.43 & 0.51 & 3.0 & 8.1 & 61.1 & 1.9 & 0.76 \\
\hline D1-5, 100-102 & 11.01 & 9.90 & 248.8 & 5.8 & 1.47 & 0.56 & 3.2 & & & & 0.77 \\
\hline D1 $-5,120-122$ & 11.21 & 10.09 & 252.1 & 5.6 & 1.46 & 0.55 & 3.1 & 9.2 & 71.0 & 2.2 & 0.68 \\
\hline D1-5, 140-142 & 11.41 & 10.30 & 256.0 & 4.5 & 1.43 & 0.50 & 2.5 & 7.6 & 57.8 & 1.4 & 0.68 \\
\hline D1-6, 20-22 & 11.71 & 10.64 & 264.3 & 3.8 & 1.42 & 0.49 & 1.8 & 9.2 & 67.7 & 1.2 & 1.04 \\
\hline D1- $6,40-42$ & 11.91 & 10.87 & 271.1 & 3.3 & 1.39 & 0.46 & 1.5 & & & & 0.88 \\
\hline D1-6, 60-62 & 12.11 & 11.07 & 277.5 & 3.1 & 1.38 & 0.44 & 1.4 & 7.8 & 58.6 & 0.8 & 0.79 \\
\hline D1-6, $80-82$ & 12.31 & 11.26 & 283.7 & 3.1 & 1.44 & 0.52 & 1.6 & 8.8 & 68.9 & 1.1 & 0.53 \\
\hline D1-6, 100-102 & 12.51 & 11.44 & 289.4 & 3.2 & 1.40 & 0.47 & 1.5 & & & & 0.78 \\
\hline D1-6, 120-122 & 12.71 & 11.62 & 295.0 & 3.2 & 1.44 & 0.52 & 1.7 & 8.3 & 63.5 & 1.1 & 0.65 \\
\hline D1-6, $140-142$ & 12.91 & 11.82 & 301.1 & 3.3 & 1.47 & 0.57 & 1.9 & 8.2 & 58.8 & 1.1 & 1.14 \\
\hline B2-3, 142-144 & 11.43 & 11.99 & 306.2 & 3.3 & 1.47 & 0.57 & 1.9 & 9.3 & 72.5 & 1.4 & 0.55 \\
\hline B $2-4,8-10$ & 11.59 & 12.15 & 311.0 & 3.3 & 1.47 & 0.57 & 1.9 & 8.8 & 67.1 & 1.3 & 0.74 \\
\hline C2-1, 93-95 & 12.94 & 12.41 & 318.9 & 3.3 & 1.41 & 0.47 & 1.6 & 8.6 & 66.2 & 1.0 & 0.63 \\
\hline $\mathrm{C} 2-1,110-112$ & 13.11 & 12.54 & 322.7 & 3.5 & 1.48 & 0.58 & 2.0 & 9.2 & 72.0 & 1.4 & 0.55 \\
\hline $\mathrm{C} 2-1,122-124$ & 13.23 & 12.69 & 327.0 & 3.9 & 1.48 & 0.57 & 2.1 & 9.6 & 75.9 & 1.6 & 0.49 \\
\hline $\mathrm{C} 2-1,140-142$ & 13.41 & 13.10 & 337.1 & 4.2 & 1.45 & 0.53 & 2.4 & 9.6 & 73.9 & 1.8 & 0.68 \\
\hline $\mathrm{C} 2-2,10-12$ & 13.61 & 13.29 & 341.2 & 4.6 & 1.46 & 0.55 & 2.5 & 10.0 & 76.2 & 1.9 & 0.85 \\
\hline $\mathrm{C} 2-2,30-32$ & 13.81 & 13.41 & 343.9 & 4.6 & 1.48 & 0.58 & 2.7 & 10.0 & 75.8 & 2.0 & 0.40 \\
\hline$C 2-2,50-52$ & 14.01 & 13.60 & 348.0 & 4.5 & 1.43 & 0.50 & 2.3 & 9.8 & 76.8 & 1.7 & 0.56 \\
\hline $\mathrm{C} 2-2,70-72$ & 14.21 & 13.80 & 352.5 & 4.4 & 1.48 & 0.58 & 2.5 & 9.9 & 77.3 & 1.9 & 0.58 \\
\hline $\mathrm{C} 2-2,90-92$ & 14.41 & 13.92 & 355.3 & 4.2 & 1.49 & 0.59 & 2.5 & 9.6 & 76.7 & 1.9 & 0.41 \\
\hline $\mathrm{C} 2-2,110-112$ & 14.61 & 14.03 & 358.0 & 4.0 & 1.47 & 0.56 & 2.3 & 9.9 & 76.1 & 1.8 & 0.80 \\
\hline $\mathrm{C} 2-2,130-132$ & 14.81 & 14.21 & 362.5 & 3.8 & 1.50 & 0.60 & 2.3 & 9.4 & 71.6 & 1.7 & 0.81 \\
\hline $\mathrm{C} 2-3,1-3$ & 15.02 & 14.52 & 371.0 & 3.5 & 1.48 & 0.58 & 2.0 & 9.4 & 74.8 & 1.5 & 0.46 \\
\hline $\mathrm{C} 2-3,23-25$ & 15.24 & 14.74 & 377.6 & 3.3 & 1.52 & 0.63 & 2.0 & 9.8 & 75.3 & 1.5 & 0.78 \\
\hline $\mathrm{C} 2-3,42-44$ & 15.43 & 14.91 & 382.9 & 3.2 & 1.49 & 0.59 & 1.9 & 10.1 & 77.7 & 1.4 & 0.50 \\
\hline$C 2-3,62-64$ & 15.63 & 15.12 & 389.6 & 3.2 & 1.48 & 0.57 & 1.8 & 9.5 & & & 0.31 \\
\hline $\mathrm{C} 2-3,83-85$ & 15.84 & 15.41 & 398.4 & 3.3 & 1.50 & 0.61 & 2.1 & 10.2 & & & 0.47 \\
\hline $\mathrm{D} 2-1,10-12$ & 13.61 & 15.45 & 399.6 & 3.5 & 1.51 & 0.62 & 2.1 & 9.3 & 73.5 & 1.6 & 0.48 \\
\hline D2-1, 30-32 & 13.81 & 15.66 & 405.6 & 3.6 & 1.53 & 0.65 & 2.3 & 9.7 & 76.6 & 1.8 & 0.55 \\
\hline D2-1, 50-52 & 14.01 & 15.88 & 411.7 & 3.6 & 1.55 & 0.68 & 2.4 & 9.6 & 75.4 & 1.8 & 0.53 \\
\hline D2-1, 70-72 & 14.21 & 16.09 & 417.5 & 3.6 & 1.54 & 0.66 & 2.4 & 9.7 & 77.0 & 1.8 & 0.44 \\
\hline D2 $-2,10-12$ & 14.54 & 16.44 & 427.3 & 3.6 & 1.59 & 0.73 & 2.6 & 9.4 & 72.9 & 1.9 & 0.63 \\
\hline D2 $-2,30-32$ & 14.74 & 16.64 & 432.9 & 3.6 & 1.54 & 0.65 & 2.4 & 10.0 & 67.0 & 1.6 & 1.79 \\
\hline
\end{tabular}


Table 1 (continued).

\begin{tabular}{|c|c|c|c|c|c|c|c|c|c|c|c|}
\hline $\begin{array}{l}\text { Core, section, } \\
\text { interval }(\mathrm{cm})\end{array}$ & $\begin{array}{l}\text { Depth } \\
\text { (mbsf) }\end{array}$ & $\begin{array}{l}\text { Rev } \\
\text { med }\end{array}$ & Age & LSR & WBD & DBD & BAR & $\begin{array}{l}\text { Mean } \\
\text { TC }\end{array}$ & $\mathrm{CaCO}_{3}$ & $\mathrm{CaCO}_{3}$-AR & $\begin{array}{l}\text { Mean } \\
\text { TOC }\end{array}$ \\
\hline D2-2, 50-52 & 14.94 & 16.85 & 438.6 & 3.8 & 1.57 & 0.70 & 2.6 & 9.9 & 76.4 & 2.0 & 0.76 \\
\hline D2-2, 70-72 & 15.14 & 17.05 & 443.7 & 4.0 & 1.55 & 0.68 & 2.7 & 10.0 & 80.1 & 2.2 & 0.35 \\
\hline D2-2, 90-92 & 15.34 & 17.24 & 448.3 & 4.4 & 1.58 & 0.71 & 3.1 & 9.8 & 79.0 & 2.5 & 0.27 \\
\hline D2-2, 110-112 & 15.54 & 17.44 & 452.6 & 4.8 & 1.55 & 0.67 & 3.2 & 9.9 & 79.7 & 2.6 & 0.37 \\
\hline D2-2, 130-132 & 15.74 & 17.64 & 456.6 & 5.3 & 1.57 & 0.70 & 3.7 & 9.6 & 77.5 & 2.9 & 0.31 \\
\hline D2-3, 10-12 & 16.04 & 17.93 & 461.9 & 5.6 & 1.54 & 0.65 & 3.7 & 7.7 & 61.7 & 2.3 & 0.24 \\
\hline D2-3, 30-32 & 16.24 & 18.13 & 465.3 & 5.9 & 1.46 & 0.55 & 3.2 & 9.0 & 73.0 & 2.4 & 0.28 \\
\hline D2 $-3,50-52$ & 16.44 & 18.33 & 468.7 & 5.7 & 1.45 & 0.54 & 3.1 & 10.1 & 80.5 & 2.5 & 0.43 \\
\hline D2 $-3,70-72$ & 16.64 & 18.54 & 472.5 & 5.4 & 1.51 & 0.61 & 3.4 & 8.8 & 70.6 & 2.4 & 0.30 \\
\hline D2-3, 90-92 & 16.84 & 18.74 & 476.3 & 5.1 & 1.51 & 0.62 & 3.1 & 8.2 & 63.1 & 2.0 & 0.58 \\
\hline D2-3, 110-112 & 17.04 & 18.94 & 480.4 & 4.6 & 1.52 & 0.63 & 2.9 & 8.1 & 63.9 & 1.8 & 0.39 \\
\hline D2-4, 10-12 & 17.18 & 19.08 & 483.7 & 4.0 & 1.52 & 0.63 & 2.6 & 7.7 & 61.1 & 1.6 & 0.34 \\
\hline D2 $-4,30-32$ & 17.38 & 19.28 & 488.8 & 3.6 & 1.49 & 0.59 & 2.1 & 9.5 & 76.3 & 1.6 & 0.30 \\
\hline D2 $-4,50-52$ & 17.58 & 19.48 & 494.7 & 3.2 & 1.52 & 0.63 & 2.0 & 9.5 & 76.2 & 1.6 & 0.37 \\
\hline D2-4, 70-72 & 17.78 & 19.68 & 501.2 & 3.0 & 1.51 & 0.61 & 1.9 & 9.0 & 72.5 & 1.3 & 0.28 \\
\hline D2-4, 90-92 & 17.98 & 19.88 & 507.9 & 3.0 & 1.50 & 0.60 & 1.8 & 8.9 & 71.4 & 1.3 & 0.34 \\
\hline D2-4, 110-112 & 18.18 & 20.08 & 514.4 & 3.1 & 1.51 & 0.62 & 1.9 & 8.4 & 67.3 & 1.3 & 0.30 \\
\hline D2 $-4,130-132$ & 18.38 & 20.28 & 520.6 & 3.6 & 1.48 & 0.57 & 2.0 & 9.0 & 73.0 & 1.5 & 0.28 \\
\hline D2-5, 10-12 & 18.59 & 20.48 & 525.6 & 4.5 & 1.38 & 0.44 & 2.1 & 8.2 & 65.3 & 1.4 & 0.33 \\
\hline D2-5, 50-52 & 18.99 & 20.88 & 534.2 & 3.2 & 1.42 & 0.50 & 1.7 & 9.4 & 68.6 & 1.1 & 0.42 \\
\hline D2-5, 70-72 & 19.19 & 21.09 & 542.2 & 2.3 & 1.52 & 0.64 & 1.4 & 8.2 & 55.4 & 0.8 & 0.61 \\
\hline D2-5, 90-92 & 19.39 & 21.29 & 552.1 & 2.1 & 1.49 & 0.59 & 1.2 & 8.6 & 67.8 & 0.8 & 0.44 \\
\hline D2-5, 110-112 & 19.59 & 21.48 & 561.0 & 2.4 & 1.44 & 0.52 & 1.3 & 6.7 & 52.8 & 0.7 & 0.36 \\
\hline D2-5, 130-132 & 19.79 & 21.70 & 569.0 & 3.1 & 1.44 & 0.52 & 1.6 & 7.0 & 53.5 & 0.9 & 0.56 \\
\hline D2-6, 10-12 & 20.10 & 22.00 & 577.8 & 3.4 & 1.43 & 0.51 & 1.8 & 7.9 & 62.6 & 1.1 & 0.41 \\
\hline D2 $-6,30-32$ & 20.29 & 22.20 & 583.5 & 3.4 & 1.46 & 0.55 & 1.9 & 8.3 & 65.4 & 1.3 & 0.45 \\
\hline D2-6, 50-52 & 20.49 & 22.39 & 589.2 & 3.1 & 1.50 & 0.60 & 1.9 & 7.5 & 57.9 & 1.1 & 0.53 \\
\hline D2-6, 70-72 & 20.69 & 22.58 & 595.7 & 2.7 & 1.47 & 0.57 & 1.5 & & & & 0.49 \\
\hline D2-6, 90-92 & 20.89 & 22.76 & 603.0 & 2.3 & 1,47 & 0.56 & 1.3 & 6.9 & 54.0 & 0.7 & 0.45 \\
\hline D2-6, 110-112 & 21.09 & 22.95 & 611.8 & 2.1 & 1.48 & 0.57 & 1.2 & 6.4 & 50.8 & 0.6 & 0.32 \\
\hline D2-6, 130-132 & 21.29 & 23.15 & 621.5 & 2.1 & 1.46 & 0.55 & 1.2 & 7.8 & 53.5 & 0.6 & 0.32 \\
\hline B3-4, 8-10 & 21.09 & 23.18 & 623.0 & 2.3 & 1.46 & 0.55 & 1.2 & 7.8 & 59.8 & 0.7 & 0.65 \\
\hline B3-4, 27-29 & 21.28 & 23.34 & 629.7 & 2.7 & 1.50 & 0.60 & 1.6 & 8.6 & 61.4 & 1.0 & 0.50 \\
\hline B3-4. 47-49 & 21.48 & 23.51 & 635.3 & 3.5 & 1.48 & 0.57 & 2.0 & 8.7 & 67.7 & 1.4 & 0.57 \\
\hline B3-4. 66-68 & 21.67 & 23.70 & 640.1 & 4.4 & 1.47 & 0.56 & 2.4 & 8.7 & 67.5 & 1.6 & 0.57 \\
\hline B3-4, 87-89 & 21.88 & 23.93 & 644.9 & 5.1 & 1.47 & 0.57 & 3.0 & 8.9 & 70.5 & 2.1 & 0.49 \\
\hline B3-4, $107-109$ & 22.08 & 24.13 & 648.5 & 5.8 & 1.46 & 0.55 & 3.2 & 8.4 & 66.0 & 2.1 & 0.51 \\
\hline B3-4, 128-130 & 22.29 & 24.38 & 652.6 & 6.1 & 1.47 & 0.55 & 3.4 & 8.7 & 68.2 & 2.3 & 0.51 \\
\hline B3-5, 0-2 & 22.51 & 24.54 & 655.2 & 5.9 & 1.46 & 0.55 & 3.3 & 8.2 & 65.0 & 2.1 & 0.36 \\
\hline B3-5, 21-23 & 22.72 & 24.75 & 658.9 & 5.2 & 1.43 & 0.50 & 2.7 & 7.6 & 60.1 & 1.6 & 0.41 \\
\hline D3-1, 110-112 & 24.11 & 25.48 & 682.3 & 1.9 & 1.43 & 0.51 & 1.0 & 7.8 & 58.6 & 0.6 & 0.76 \\
\hline D3-1, 130-132 & 24.31 & 25.55 & 686.2 & 1.9 & 1.44 & 0.53 & 0.9 & 8.2 & 63.5 & 0.6 & 0.60 \\
\hline D3-2, 10-12 & 24.61 & 26.02 & 710.2 & 2.4 & 1.45 & 0.54 & 1.5 & 7.3 & 56.3 & 0.8 & 0.53 \\
\hline D3-2, 30-32 & 24.81 & 26.35 & 720.0 & 3.5 & 1.44 & 0.52 & 2.0 & 7.7 & 57.5 & 1.1 & 0.75 \\
\hline D3-2, 50-52 & 25.01 & 26.46 & 722.9 & 3.9 & 1.47 & 0.56 & 2.2 & 7.4 & 55.5 & 1.2 & 0.71 \\
\hline D3-2, 70-72 & 25.21 & 26.60 & 726.4 & 3.9 & 1.47 & 0.56 & 2.2 & & & & 0.40 \\
\hline D3-2, 90-92 & 25.41 & 27.10 & 739.5 & 3.7 & 1.44 & 0.52 & 1.9 & 7.3 & 55.5 & 1.1 & 0.61 \\
\hline D3-2, $110-112$ & 25.61 & 27.39 & 747.6 & 3.6 & 1.46 & 0.55 & 2.0 & 6.8 & 51.2 & 1.0 & 0.61 \\
\hline D3-2, 130-132 & 25.81 & 27.51 & 750.9 & 3.7 & 1.44 & 0.52 & 1.9 & 7.4 & 55.6 & 1.0 & 0.77 \\
\hline D3-3, 10-12 & 26.11 & 27.77 & 757.9 & 3.8 & 1.42 & 0.49 & 1.9 & 5.6 & 40.9 & 0.8 & 0.68 \\
\hline D3-3, $30-32$ & 26.31 & 28.01 & 764.0 & 4.1 & 1.36 & 0.41 & 1.7 & 4.1 & 28.3 & 0.5 & 0.68 \\
\hline D3-3, 50-52 & 26.51 & 28.22 & 769.0 & 4.3 & 1.36 & 0.41 & 1.8 & 4.8 & 34.0 & 0.6 & 0.73 \\
\hline D3-3, 70-72 & 26.71 & 28.41 & 773.3 & 4.5 & 1.40 & 0.46 & 2.1 & 5.7 & 42.0 & 0.9 & 0.62 \\
\hline D3-3, 90-92 & 26.91 & 28.61 & 777.7 & 4.6 & 1.47 & 0.56 & 2.6 & 7.8 & 61.9 & 1.6 & 0.41 \\
\hline D3-3, $110-112$ & 27.11 & 28.81 & 782.0 & 4.8 & 1.51 & 0.61 & 2.9 & 8.5 & 67.5 & 2.0 & 0.43 \\
\hline D3-3, 130-132 & 27.31 & 29.02 & 786.2 & 5.3 & 1.47 & 0.56 & 2.9 & 8.0 & 63.6 & 1.8 & 0.36 \\
\hline D3-4, 11-13 & 27.62 & 29.31 & 791.5 & 5.4 & 1.45 & 0.53 & 2.9 & 6.5 & 50.1 & 1.5 & 0.46 \\
\hline D3-4, 31-33 & 27.82 & 29.52 & 795.4 & 5.3 & 1.46 & 0.54 & 2.9 & 6.0 & 44.8 & 1.3 & 0.40 \\
\hline D3-4, $51-53$ & 28.02 & 29.73 & 799.5 & 4.9 & 1.45 & 0.53 & 2.6 & 6.2 & 48.4 & 1.3 & 0.40 \\
\hline D3-4, 71-73 & 28.22 & 29.91 & 803.3 & 4.6 & 1.46 & 0.54 & 2.5 & 7.7 & 61.0 & 1.5 & 0.40 \\
\hline D3-4, 91-93 & 28.42 & 30.12 & 808.0 & 4.3 & 1.41 & 0.48 & 2.0 & 8.2 & 64.9 & 1.3 & 0.37 \\
\hline D3-4, 111-113 & 28.62 & 30.32 & 812.9 & 3.9 & 1.41 & 0.48 & 1.9 & & & & 0.50 \\
\hline D3-4, 131-133 & 28.82 & 30.51 & 818.0 & 3.5 & 1.40 & 0.47 & 1.7 & 7.4 & 58.0 & 1.0 & 0.42 \\
\hline D3-5, 10-12 & 29.11 & 30.81 & 826.8 & 3.3 & 1.40 & 0.47 & 1.5 & 5.8 & 44.8 & 0.7 & 0.46 \\
\hline D3-5, 30-32 & 29.31 & 31.01 & 833.3 & 3.0 & 1.39 & 0.45 & 1.3 & 5.2 & 39.8 & 0.5 & 0.43 \\
\hline D3-5, 50-52 & 29.51 & 31.21 & 840.2 & 2.9 & 1.45 & 0.53 & 1.5 & 7.9 & 61.6 & 0.9 & 0.50 \\
\hline D3-5, 70-72 & 29.71 & 31.41 & 847.2 & 2.9 & 1.41 & 0.48 & 1.4 & 6.1 & 46.7 & 0.6 & 0.49 \\
\hline D3-5, $110-112$ & 30.11 & 31.81 & 860.8 & 3.1 & 1.46 & 0.55 & 1.7 & 6.9 & 54.0 & 0.9 & 0.43 \\
\hline D3-5, $130-132$ & 30.31 & 32.05 & 868.0 & 3.7 & 1.46 & 0.55 & 1.9 & 8.0 & 63.3 & 1.2 & 0.44 \\
\hline D3-6, 10-12 & 30.61 & 32.49 & 879.4 & 4.0 & 1.49 & 0.59 & 2.5 & 9.3 & 73.4 & 1.8 & 0.45 \\
\hline D3-6, 30-32 & 30.81 & 32.68 & 883.8 & 4.4 & 1.45 & 0.54 & 2.4 & 8.8 & 69.3 & 1.6 & 0.48 \\
\hline D3-6, 50-52 & 31.01 & 32.84 & 887.4 & 4.5 & 1.47 & 0.56 & 2.5 & 9.1 & 71.9 & 1.8 & 0.43 \\
\hline D3-6, 70-72 & 31.21 & 33.05 & 892.0 & 4.5 & 1.48 & 0.58 & 2.6 & 8.9 & 71.1 & 1.9 & 0.40 \\
\hline D3-6, 90-92 & 31.41 & 33.28 & 897.1 & 4.6 & 1.50 & 0.60 & 2.7 & 9.6 & 76.6 & 2.1 & 0.38 \\
\hline D3-6, 110-112 & 31.61 & 33.41 & 899.9 & 4.9 & 1.49 & 0.58 & 2.9 & 8.5 & 68.0 & 2.0 & 0.35 \\
\hline D3-6, 130-132 & 31.81 & 33.50 & 901.6 & 5.9 & 1.47 & 0.56 & 3.0 & 8.7 & 70.5 & 2.1 & 0.24 \\
\hline D3-7, 10-12 & 32.11 & 33.74 & 905.5 & 6.1 & 1.48 & 0.58 & 3.9 & 7.8 & 61.1 & 2.4 & 0.42 \\
\hline D3-7, 30-32 & 32.31 & 34.17 & 912.5 & 5.5 & 1.49 & 0.59 & 3.0 & 8.9 & 69.4 & 2.1 & 0.53 \\
\hline D3-7, 50-52 & 32.51 & 34.38 & 917.1 & 4.1 & 1.46 & 0.55 & 2.2 & 8.2 & 65.0 & 1.4 & 0.35 \\
\hline D3-7, 70-72 & 32.71 & 34.58 & 922.5 & 3.5 & 1.42 & 0.50 & 1.7 & 7.0 & 54.5 & 0.9 & 0.42 \\
\hline$C 4-1,48-50$ & 31.49 & 34.69 & 925.9 & 3.0 & 1.40 & 0.46 & 1.4 & 7.1 & 56.7 & 0.8 & 0.29 \\
\hline
\end{tabular}


Table 1 (continued).

\begin{tabular}{|c|c|c|c|c|c|c|c|c|c|c|c|c|c|c|c|c|}
\hline $\begin{array}{l}\text { Core, section, } \\
\text { interval }(\mathrm{cm})\end{array}$ & $\begin{array}{l}\text { Depth } \\
\text { (mbsf) }\end{array}$ & $\begin{array}{l}\operatorname{Rev} \\
\text { med }\end{array}$ & Age & LSR & WBD & DBD & BAR & $\begin{array}{l}\text { Mean } \\
\text { TC }\end{array}$ & $\mathrm{CaCO}_{3}$ & $\mathrm{CaCO}_{3}-\mathrm{AR}$ & $\begin{array}{l}\text { Mean } \\
\text { TOC }\end{array}$ & $\pm 1 \sigma$ & TOC-AR & $\mathrm{U}_{37}^{\mathrm{K}}$ & SST & $\pm 1 \sigma$ \\
\hline C4-1,89-91 & 31.90 & 35.11 & 941.4 & 2.5 & 1.49 & 0.58 & 1.4 & 8.2 & 66.5 & 1.0 & 0.21 & 0.00 & 2.9 & 0.884 & 24.9 & \\
\hline $\mathrm{C} 4-1,110-112$ & 32.11 & 35.31 & 949.6 & 2.6 & 1.52 & 0.63 & 1.6 & 8.5 & 69.2 & 1.1 & 0.19 & 0.02 & 3.1 & 0.806 & 22.6 & \\
\hline $\mathrm{C} 4-1,130-132$ & 32.31 & 35.51 & 956.6 & 3.2 & 1.50 & 0.60 & 2.0 & 8.9 & 70.3 & 1.4 & 0.37 & 0.03 & 7.3 & 0.877 & 24.6 & 0.21 \\
\hline$C 4-2,2-4$ & 32.53 & 35.73 & 962.6 & 3.7 & 1.46 & 0.55 & 2.1 & 8.5 & 68.6 & 1.4 & 0.27 & 0.01 & 5.7 & 0.856 & 24.0 & \\
\hline $\mathrm{C} 4-2,22-24$ & 32.73 & 35.93 & 968.1 & 3.4 & 1.50 & 0.60 & 2.0 & 9.0 & 73.4 & 1.5 & 0.21 & 0.05 & 4.3 & 0.859 & 24.1 & \\
\hline $\mathrm{C} 4-2,42-44$ & 32.93 & 36.13 & 974.5 & 2.9 & 1.50 & 0.61 & 1.7 & 8.8 & 71.7 & 1.3 & 0.22 & 0.01 & 3.8 & 0.847 & 23.8 & \\
\hline D4-1, 10-12 & 32.61 & 36.32 & 981.7 & 2.6 & 1.49 & 0.60 & 1.4 & 8.2 & 65.9 & 1.0 & 0.27 & 0.02 & 3.8 & 0.816 & 22.8 & 0.50 \\
\hline C4-2, 62-64 & 33.13 & 36.33 & 982.2 & 2.2 & 1.50 & 0.60 & 1.4 & & & & 0.24 & 0.00 & 3.4 & 0.859 & 24.1 & \\
\hline D4-1, 30-32 & 32.81 & 36.52 & 990.7 & 2.2 & 1.53 & 0.65 & 1.3 & 7.1 & 56.7 & 0.8 & 0.31 & 0.01 & 4.2 & 0.821 & 23.0 & 0.07 \\
\hline C $4-2,83-85$ & 33.34 & 36.54 & 991.7 & 1.9 & 1.48 & 0.58 & 1.2 & & & & 0.23 & 0.04 & 2.6 & 0.867 & 24.4 & 0.00 \\
\hline D4-1, 50-52 & 33.01 & 36.70 & 1000.0 & 1.8 & 1.49 & 0.59 & 1.1 & 8.1 & 64.0 & 0.7 & 0.37 & 0.01 & 4.0 & & & \\
\hline D4-1, 70-72 & 33.21 & 36.90 & 1012.0 & 1.7 & 1.51 & 0.61 & 1.0 & 7.9 & 63.1 & 0.7 & 0.31 & 0.06 & 3.2 & 0.794 & 22.2 & \\
\hline D4-1, $90-92$ & 33.41 & 37.09 & 1023.0 & 1.9 & 1.50 & 0.60 & 1.1 & 8.4 & 68.1 & 0.7 & 0.25 & 0.01 & 2.7 & 0.831 & 23.3 & \\
\hline D4-1, 110-112 & 33.61 & 37.29 & 1033.0 & 2.2 & 1.55 & 0.67 & 1.4 & 6.5 & 51.7 & 0.7 & 0.24 & 0.04 & 3.5 & 0.849 & 23.8 & \\
\hline D4-1, $130-132$ & 33.81 & 37.49 & 1041.0 & 3.1 & 1.50 & 0.61 & 1.8 & 6.8 & 54.1 & 1.0 & 0.26 & 0.01 & 4.7 & 0.843 & 23.6 & \\
\hline D $4-2,10-12$ & 34.11 & 37.78 & 1049.0 & 4.0 & 1.52 & 0.63 & 2.8 & 7.3 & 58.3 & 1.7 & 0.34 & 0.02 & 9.6 & 0.817 & 22.9 & 0.07 \\
\hline D4-2, 30-32 & 34.31 & 37.97 & 1053.0 & 4.9 & 1.52 & 0.63 & 3.3 & 7.7 & 60.9 & 2.0 & 0.37 & 0.00 & 12.3 & 0.791 & 22.1 & \\
\hline D4-2, 50-52 & 34.51 & 38.17 & 1057.0 & 5.6 & 1.52 & 0.63 & 3.6 & 7.9 & 61.2 & 2.2 & 0.58 & 0.05 & 20.9 & & & \\
\hline D $4-2,70-72$ & 34.71 & 38.36 & 1060.0 & 6.7 & 1.50 & 0.61 & 3.7 & 6.0 & 47.3 & 1.8 & 0.30 & 0.01 & 11.3 & 0.819 & 22.9 & \\
\hline D4-2, $90-92$ & 34.91 & 38.57 & 1063.0 & 6.5 & 1.46 & 0.55 & 3.5 & 6.3 & 50.2 & 1.8 & 0.32 & 0.06 & 11.2 & 0.814 & 22.8 & \\
\hline $\mathrm{D} 4-2,110-112$ & 35.11 & 38.75 & 1066.0 & 6.3 & 1.48 & 0.57 & 3.7 & 6.8 & 53.8 & 2.0 & 0.33 & 0.08 & 12.1 & 0.861 & 24.2 & 0.07 \\
\hline D4-2, 130-132 & 35.31 & 38.95 & 1069.0 & 6.3 & 1.46 & 0.55 & 3.4 & 7.8 & 61.5 & 2.1 & 0.42 & 0.01 & 14.4 & 0.788 & 22.0 & \\
\hline D4-3, $10-12$ & 35.61 & 39.25 & 1074.0 & 5.7 & 1.45 & 0.54 & 3.0 & 6.6 & 52.2 & 1.6 & 0.29 & 0.01 & 8.8 & 0.846 & 23.7 & 0.85 \\
\hline D4-3, 30-32 & 35.81 & 39.46 & 1078.0 & 5.1 & 1.45 & 0.53 & 2.7 & 6.8 & 54.6 & 1.5 & 0.29 & 0.02 & 7.8 & 0.878 & 24.7 & \\
\hline D4-3, 50-52 & 36.01 & 39.66 & 1082.0 & 4.6 & 1.46 & 0.55 & 2.6 & 7.4 & 59.0 & 1.5 & 0.30 & 0.01 & 7.5 & & & \\
\hline D $4-3,70-72$ & 36.21 & 39.87 & 1087.0 & 4.4 & 1.48 & 0.57 & 2.4 & 7.6 & 59.5 & 1.5 & 0.37 & 0.07 & 9.0 & 0.841 & 23.6 & 0.21 \\
\hline D4-3, 90-92 & 36.41 & 40.10 & 1092.0 & 3.9 & 1.41 & 0.48 & 1.9 & 5.0 & 38.5 & 0.7 & 0.36 & 0.02 & 6.9 & 0.792 & 22.1 & \\
\hline D4-3, $110-112$ & 36.61 & 40.34 & 1099.0 & 3.6 & 1.39 & 0.45 & 1.7 & 5.8 & 44.8 & 0.8 & 0.41 & 0.00 & 7.0 & & & \\
\hline D4-3, $130-132$ & 36.81 & 40.53 & 1104.0 & 4.0 & 1.42 & 0.50 & 1.9 & 6.1 & 47.5 & 0.9 & 0.40 & 0.01 & 7.6 & 0.832 & 23.3 & \\
\hline D $4-4,10-12$ & 37.11 & 40.74 & 1109.0 & 3.9 & 1.45 & 0.54 & 2.1 & 7.4 & 59.3 & 1.3 & 0.26 & 0.03 & 5.5 & 0.863 & 24.2 & 0.07 \\
\hline D4 $4,30-32$ & 37.31 & 40.88 & 1113.0 & 3.9 & 1.44 & 0.52 & 0.8 & 7.6 & 61.4 & 0.5 & 0.24 & 0.02 & 2.0 & 0.850 & 23.9 & \\
\hline D4-4, 50-52 & 37.51 & 41.05 & 1117.0 & 4.1 & 1.43 & 0.50 & 2.1 & 7.1 & 56.0 & 1.2 & 0.36 & 0.14 & 7.5 & & & \\
\hline D $4-4,70-72$ & 37.71 & 41.25 & 1122.0 & 4.2 & 1.42 & 0.50 & 2.1 & 6.5 & 51.0 & 1.1 & 0.41 & 0.02 & 8.7 & 0.816 & 22.9 & \\
\hline D4-4, $90-92$ & 37.91 & 41.51 & 1128.0 & 4.5 & 1.42 & 0.50 & 2.1 & 6.6 & 49 . & 1.0 & 0.67 & 0.0 & 14.2 & 0.804 & 22.5 & \\
\hline D4-4, $110-112$ & 38.11 & 41.75 & 1133.0 & 4.4 & 1.42 & 0.50 & 2.1 & 6.4 & 49.1 & 1.0 & 0.4 & 0.1 & 10.2 & 0.855 & 24.0 & \\
\hline D4-4, 130-132 & 38.31 & 41.95 & 1138.0 & 3.9 & 1.38 & 0.44 & 1.8 & 5.1 & 38.9 & 0.7 & 0.47 & 0.03 & 8.6 & 0.846 & 23.7 & 0.28 \\
\hline D4-5, 10-12 & 38.61 & 42.22 & 1145.0 & 4.2 & 1.38 & 0.44 & 1.8 & 6.7 & 52.4 & 0.9 & 0.58 & 0.07 & 10.5 & 0.840 & 23.6 & \\
\hline D4-5, 30-32 & 38.81 & 42.41 & 1149.0 & 4.3 & 1.43 & 0.51 & 2.1 & 7.1 & 55.1 & 1.2 & 0.45 & 0.1 & 9.5 & 0.818 & 22.9 & \\
\hline D4-5, 50-52 & 39.01 & 42.61 & 1154.0 & 4.1 & 1.43 & 0.51 & 2.2 & 7.1 & 55. & 1.2 & 0.46 & 0.00 & 10.1 & & & \\
\hline D4-5, 70-72 & 39.21 & 42.82 & 1159.0 & 4.7 & 1.42 & 0.50 & 2.2 & 6.2 & 47.0 & 1.0 & 0.56 & 0.02 & 12.4 & 0.853 & 23.9 & \\
\hline D4-5, $90-92$ & 39.41 & 43.03 & 1163.0 & 5.0 & 1.48 & 0.57 & 2.6 & 6.9 & 54.4 & 1.4 & 0.41 & 0.0 & 10.8 & 0.867 & 24.4 & \\
\hline D4-5, $100-102$ & 39.51 & 43.1 & 1165 & 4.7 & 1.46 & 0.54 & 2.5 & & 37.4 & & 0.35 & 0.0 & 9.0 & 0.8 & 24.5 & \\
\hline D4-5, $120-122$ & 39.71 & 43.31 & 1169.0 & 4.9 & 1.48 & 0.58 & 2.8 & 7.9 & 61.9 & 1.7 & 0.42 & 0.01 & 11.7 & 0.957 & 27.0 & 0.35 \\
\hline D4-5, 140-142 & 39.91 & 43.51 & 1173.0 & 4.6 & 1.50 & 0.60 & 2.8 & 8.0 & & 1.8 & 0.39 & 0.07 & 11.1 & 0.927 & 26.1 & \\
\hline D4-6, 10-12 & 40.11 & 43.72 & 1178.0 & 4.1 & 1.48 & 0.58 & 2.6 & 8.4 & 67.1 & 1.8 & 0.36 & 0.10 & 9.4 & 0.896 & 25.2 & \\
\hline D4-6, 30-32 & 40.31 & 43.92 & 1183 & 3.9 & 1.49 & 0.5 & 2.4 & 8.3 & 65 & 1.6 & 0.4 & 0.11 & 9.9 & 0.918 & 25.9 & \\
\hline D4-6, 50-52 & 40.51 & 44.11 & 1188.0 & 3.7 & 1.49 & 0.59 & 2.1 & 10.5 & 83.5 & 1.7 & 0.44 & 0.08 & 9.3 & & & \\
\hline D4-6, 70-72 & 40.71 & 44.29 & 1193.0 & 3.1 & 1.47 & 0.57 & 1.7 & 8.7 & 68.7 & 1.1 & 0.44 & 0.01 & 7.3 & 0.851 & 23.9 & \\
\hline D4-6, $90-92$ & 40.91 & 44.48 & 1200 & 2.4 & 1.4 & 0. & 1.3 & 8.0 & & 0.8 & 0. & & 6.7 & 0.820 & 23.0 & \\
\hline D4-6, 110-112 & 41.11 & 44.6 & 1210 & 2.0 & 1.36 & 0. & 0.8 & 5.6 & 40 & 0.3 & 0.7 & 0. & 6.4 & 0.863 & 24.2 & 0.21 \\
\hline D4-6, $130-132$ & 41.31 & 44.91 & 1221.0 & 2.1 & 1.46 & 0.55 & 1.1 & 7.1 & 54.9 & 0.6 & 0.48 & 0.07 & 5.5 & 0.847 & 23.8 & \\
\hline D4-6, $148-150$ & 41.49 & 45.10 & 1230.0 & 2.3 & 1.48 & 0.58 & 1.4 & 7.1 & 56.3 & 0.8 & 0.30 & 0.08 & 4.2 & 0.923 & 26.0 & \\
\hline D4-7, $10-12$ & 41.61 & 45.21 & 1234.0 & 2.9 & 1.48 & 0.57 & 1.5 & 7.2 & 56.6 & 0.9 & 0.37 & 0.05 & 5.7 & 0.906 & 25.5 & \\
\hline D4-7, 30-32 & 41.81 & 45.39 & 1240 & 3.4 & 1.45 & 0. & 1.7 & 7.3 & 57.0 & 1.0 & 0.4 & 0.06 & 7.4 & 0.910 & 25.6 & \\
\hline D4-7, 50-52 & 42.01 & 45.58 & 1245.0 & 3.7 & 1.43 & 0.51 & 1.9 & 8.0 & 61.1 & 1.2 & 0.64 & 0.08 & 12.2 & & & \\
\hline D4-7, 70-72 & 42.21 & 45.80 & 1251.0 & 3.7 & 1.41 & 0.48 & 2.0 & 7.8 & 58.9 & 1.2 & 0.76 & 0.04 & 15.7 & 0.814 & 22.8 & \\
\hline
\end{tabular}

Notes: ODP depth in meters below seafloor; rev med: revised composite depth (Hagelberg et al., this volume); Age: age assignment (in Ka) for each sample midpoint based on Mix et al. (this volume); LSR: linear sedimentation rate (in cm/k.y.); WBD: GRAPE wet-bulk-density ( $\left./ \mathrm{cm}^{3}\right)$; DBD: calculated dry-bulk density (in g/cm ); BAR: Bulk accumulation rate (in $\mathrm{g} / \mathrm{cm}^{2} \mathrm{k} . \mathrm{y}^{-1}$ ); Mean TC: average wt.\% total carbon; $\mathrm{CaCO}_{3}$ : wt.\% $\mathrm{CaCO}_{3} ; \mathrm{CaCO}_{3}-\mathrm{AR}$ : calcium carbonate accumulation rate (g/cm ${ }^{2} \mathrm{k} . \mathrm{y}$. ${ }^{-1}$ ); $\mathrm{Mean}$ TOC: average wt.\% organic carbon; TOC-AR: organic carbon accumulation rate $\left(\mathrm{mg} / \mathrm{cm}^{2} \mathrm{k} . \mathrm{y} .{ }^{-1}\right)$; SST: sea-surface temperatures $\left({ }^{\circ} \mathrm{C}\right)$ calculated from $\mathrm{U}_{37}^{\mathrm{K}}$ indexes.

the weight percentage data for organic carbon and $\mathrm{CaCO}_{3}$, the estimated DBD, and the $\mathrm{U}_{37}^{\mathrm{k}}$ index plotted vs. composite depth. Organic carbon concentrations increase from the seafloor gradually to $1.5 \%$ at around $1 \mathrm{mcd}$ and slowly decrease to values less than $0.5 \%$ at about $20 \mathrm{mcd}$. The gradual decline in TOC concentrations is irregular, and concentrations vary between $2 \%$ and $0.5 \%$. Below $20 \mathrm{mcd}$, variability is lower and values fluctuate from $0.5 \%$ to $1 \%$. The $\mathrm{CaCO}_{3}$ concentrations decrease from the youngest samples near the sediment/water interface (75\%) to $5 \mathrm{mcd}(30 \%)$, increase slowly to $80 \%$ at $15 \mathrm{mcd}$, and again decrease to $<30 \%$ at 27 mcd. Below 15 mcd, the $\mathrm{CaCO}_{3}$ record varies more strongly than above. We note a strong dependence of DBD on calcium carbonate content; DBD traces both the general curve of carbonate and fluctuations on smaller scales. Decreasing densities in samples from 15 to $3 \mathrm{mcd}$ are attributable to an increase in the diatom content ("Site 846" chapter in Mayer, Pisias, Janecek, et al., 1992), which lowers the sediment density and dilutes $\mathrm{CaCO}_{3}$ concentrations. High $\mathrm{SiO}_{2}$ concentrations correlate negatively with
$\mathrm{CaCO}_{3}$ and do not correlate with $\mathrm{Al}_{2} \mathrm{O}_{3}$ concentrations in the uppermost cores at Site 846 (Torres, pers. comm., 1993).

$\mathrm{U}_{37}^{\mathrm{k}}$ indexes plotted vs. depth fluctuate between 0.7 (which corresponds to coldest SST estimates) and 1 (which corresponds to warmest SST estimates) over our record. The interval between 20 and 15 mcd, corresponding to highest calcium carbonate concentrations, marks a transition from a cooling to a warming trend toward our youngest samples. The warming trend underlies highly variable $U_{37}^{k}$ indexes in the upper record section. It tracks an increase in TOC concentrations, a decrease in $\mathrm{CaCO}_{3}$ concentrations, and a decrease in DBDs attributed to an increase in opal concentrations.

\section{Accumulation Rates and SSTs}

Estimates of accumulation rates rely primarily on linear sedimentation rates that are based on the stable oxygen isotope record of benthic foraminifers. Sedimentation rates were constrained to vary 


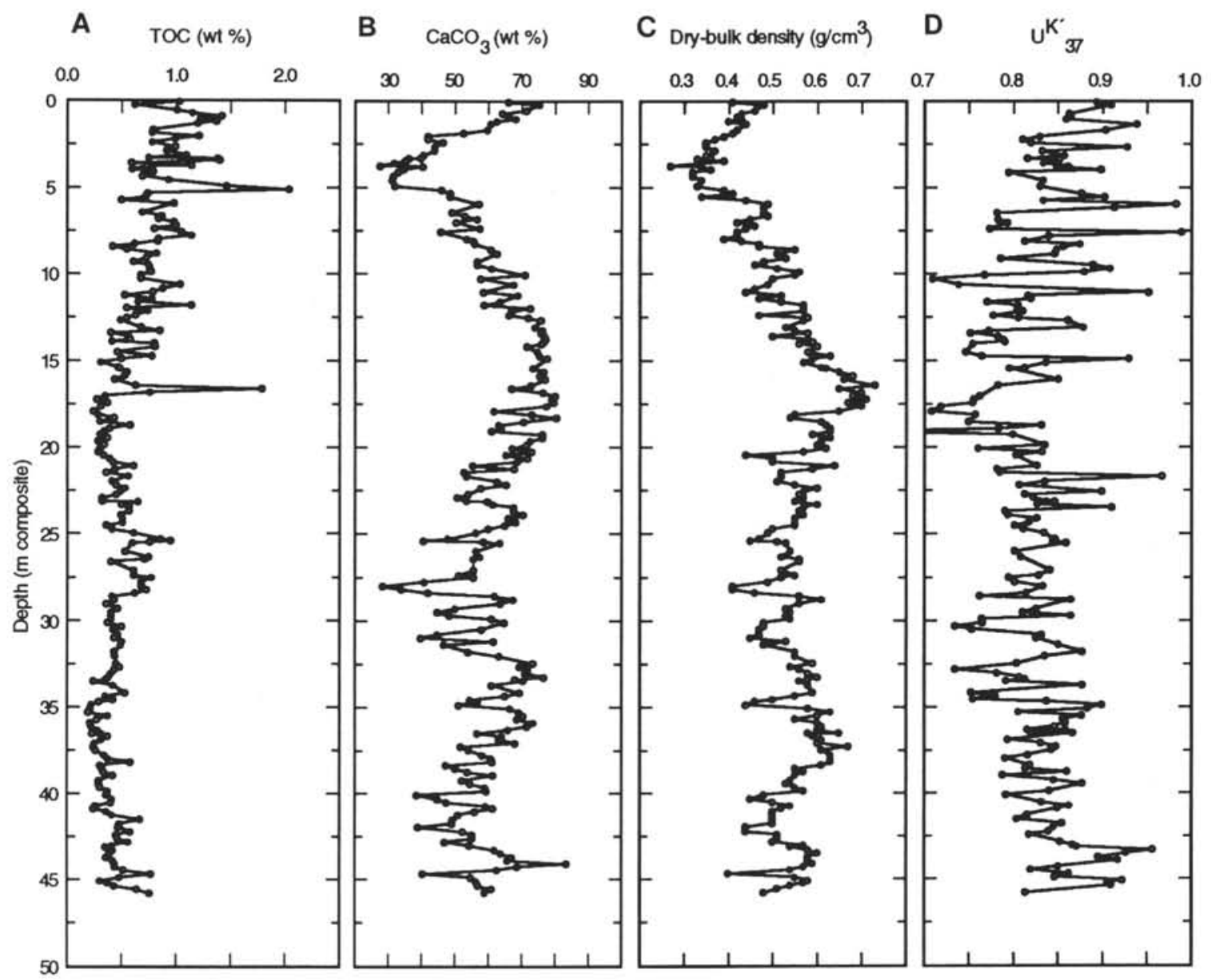

Figure 3. A. Concentrations of organic carbon. B. Concentrations of calcium carbonate. C. DBD estimates. D. $\mathrm{U}_{37}^{\mathrm{k}}$ indexes in the top 46 $\mathrm{m}$ revised depth in the sediment at Site 846 .

smoothly (Mix et al., this volume), and variance of LSR and accumulation rates at periodicities shorter than 40 k.y. may have been suppressed. Our accumulation rate calculations are further based on estimated sediment DBDs in the composite section, not on actual measurements of grain densities. In consequence, they must be regarded as preliminary.

The percentage data of $\mathrm{CaCO}_{3}$ and TOC at Site 846 translate into accumulation rate (AR) curves of considerable variability (Fig. 4). $\mathrm{CaCO}_{3}$-ARs in the older section (from 1300 to $500 \mathrm{Ka}$ and equivalent to $20 \mathrm{mcd}$ ), vary between 0.5 and $2.5 \mathrm{~g} / \mathrm{cm}^{2} \mathrm{k} . \mathrm{y} .{ }^{-1}$ without any underlying trend. After a maximum of $\mathrm{CaCO}_{3}$-accumulation between 400 and $500 \mathrm{Ka}$ ( 2 to $3 \mathrm{~g} / \mathrm{cm}^{2} \mathrm{k} \cdot \mathrm{y}^{-1}$.), the rates decrease significantly to lowest rates $\left(0.2 \mathrm{mg} / \mathrm{cm}^{2} \mathrm{k} . \mathrm{y}^{-1}\right)$ at $100 \mathrm{Ka}$. This decrease reflects the decline in concentrations from 15 to $5 \mathrm{mcd}$. Pronounced fluctuations related to glacial/interglacial cycles (depicted in the $\delta^{18} \mathrm{O}$ record) characterize the entire record. These fluctuations are most pronounced in the lower section of the record to $500 \mathrm{Ka}$, but are also superimposed on the decreasing trend after this time.

TOC-ARs in the record segment older than $500 \mathrm{Ka}$ range from 3 to $20 \mathrm{mg} / \mathrm{cm}^{2} \mathrm{k} . \mathrm{y}^{-1}$. After this time, the time series of TOC accumulation has a clear trend toward higher rates in younger samples. In addition, the rates of TOC burial show increased scatter since $480 \mathrm{Ka}$, with high variability (between 40 and $5 \mathrm{mgC} / \mathrm{cm}^{2} \mathrm{k} . \mathrm{y} .{ }^{-1}$ ) lasting into the Holocene.

In a series of cores spanning the entire central and eastern equatorial Pacific Ocean from the Holocene to $300 \mathrm{Ka}$, Lyle et al. (1988) noted intense maxima of TOC accumulation at periods centered at about 18,150 , and $280 \mathrm{Ka}$. The maximum in isotope stage 2 is not well represented in our record and blends into a period of high TOC accumulation during isotope stages 2 through 4, but we can confirm that the maxima at 150 and $250 \mathrm{Ka}$ are among the strongest and best defined in the entire period since $1300 \mathrm{Ka}$. Both are accompanied by high rates in carbonate accumulation.

Calcite accumulation rates in core tops are between 0.9 and $1.2 \mathrm{~g}$ $\mathrm{CaCO}_{3} / \mathrm{cm}^{2} \mathrm{k.y} .^{-1}$ and TOC accumulation rates are about $10 \mathrm{mg} / \mathrm{cm}^{2}$ k.y. ${ }^{-1}$ in the vicinity of Site 846 (Lyle, 1992). Comparing the accumulation rate records with the record of $\delta^{18} \mathrm{O}$ in benthic foraminifers (Fig. 4), we noted a good match in the timing of maxima in accumulation of biogenic material with increases in $\delta^{18} \mathrm{O}$ during glacials. Both carbonate and organic carbon accumulated at more than twice their modern rates and those of interglacials in glacial stages. We also noted that an increase in the amplitude of the isotope record between 500 and $400 \mathrm{Ka}$ coincides with the maximum of carbonate burial and the onset of high variability in TOC accumulation rates. Preliminary data indicate that this time coincides with an increase in burial of opal in the sediments at Site 846 (Torres, pers. comm., 1993).

SSTs at Site 846 varied between $27^{\circ}$ and $20^{\circ} \mathrm{C}$ in the entire record (Fig. 4). Short-term temperature variability was considerable and appears to modulate long-term trends. We note that SSTs decreased from an average of $24^{\circ} \mathrm{C}$ in the deepest part of our record to approximately $500 \mathrm{Ka}\left(22^{\circ} \mathrm{C}\right)$, decreased to values below $20^{\circ} \mathrm{C}$ at $480 \mathrm{Ka}$, and increased from that time to values of approximately $25^{\circ} \mathrm{C}$ in our youngest samples. Temperature variability in the lower record section is less than that in the upper portion, except for a phase of high variability in the period from 1000 to $800 \mathrm{Ka}$. During the transition period between the two trends, between 500 and $400 \mathrm{Ka}$, cold temperatures coincide with pronounced maxima in the accumulation of biogenic material, most notably of carbonate. In the upper portion of 


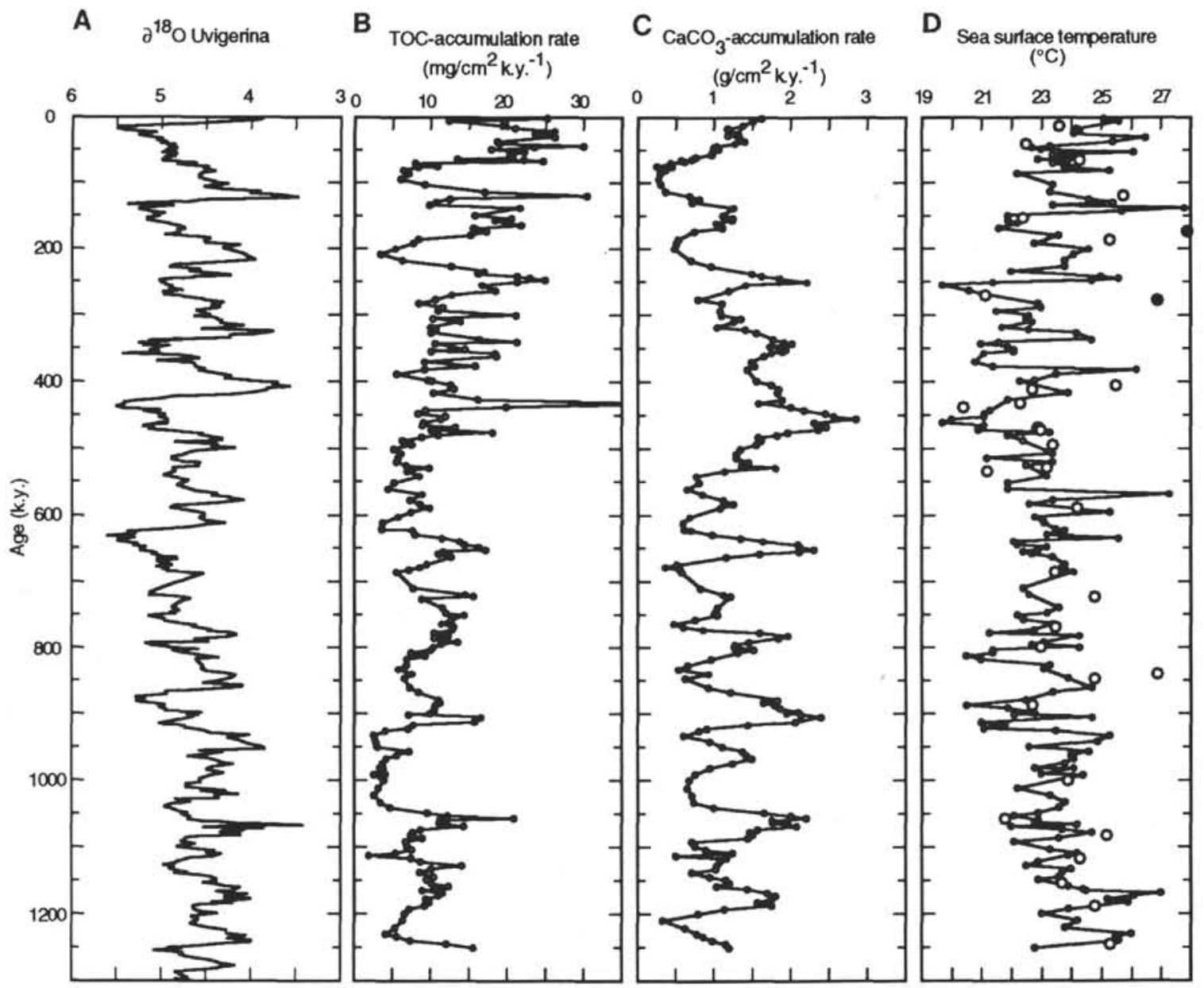

Figure 4. A. The benthic $\delta^{18} \mathrm{O}$ (Mix et al., this volume) record. B. Accumulation rates of organic carbon. C. Carbonate accumulation rates. D. SST calculated from $\mathrm{U}_{37}^{\mathrm{k}}$ indexes at Site 846 plotted vs. time. In the SST curve, filled circles represent data points that were rejected and not included in the cross-spectral analyses. Circles are temperature estimates based on measurements of $\mathrm{U}_{37}^{\mathrm{k}}$ on board JOIDES Resolution during Leg 138 .

the record, variability in SST is high (over a range of $7^{\circ} \mathrm{C}$ ) and displays patterns of glacial low and interglacial high SSTs.

We realize that the temperature calibration of the $U_{37}^{k}$ index, which is based on the genus E. huxleyi, is likely to be correct only for the last 260 k.y., corresponding to a depth of approximately $10 \mathrm{mcd}$ at Site 846. The long trend to decreasing temperatures in the lower portion of our record may be a reflection of species change that contributed to the alkenone signal, which results in a drift of the $U_{37}^{k}$. However, Marlowe et al. (1990) showed that there is reason to believe that the metabolic function of the alkenones as cell-wall lipids extends much farther into the phylogenetic lineage of the prymnesiophytes. Based on the correlation between low unsaturation indexes and high $\mathrm{CaCO}_{3}$ concentration in the period between 400 and $500 \mathrm{Ka}$, corresponding to a depth interval between 20 and $15 \mathrm{mcd}$, we can argue that the temperature low may indeed indicate colder temperatures in surface water, and that this water at this time was more productive for prymnesiophytes (coccolithophores). We see this as an indication that the inflection of the temperature record is not related to changes in the metabolism of alkenone-producing organisms.

\section{DISCUSSION}

\section{Long-term Trends}

East-west elongated contours of biogenic accumulation rates in modern sediments (Lyle, 1992) and of SSTs reconstructed from $U_{37}^{k}$ ratios in surface sediments (Fluegge, unpubl. data) depict zonal gra- dients that are weak compared to the steep north-south gradients. Back-tracking the relative plate motion of Nazca Plate showed that Site 846 moved only approximately $92 \mathrm{~km}$ due east and parallel to the prevailing direction of modern accumulation rate contours during the 1.3 m.y. covered by our record (Mayer, Pisias, Janecek, et al., 1992). The strong change in the character of accumulation rates and SST curves between 500 and $400 \mathrm{Ka}$ thus are unlikely to result from site wandering underneath any permanent hydrographic fronts at the sea surface; they most probably represent a change in long-term conditions at the sea surface over the site.

The record segment of temperature inversion to higher temperatures, coupled with a change in accumulation rate patterns (increasing TOC and decreasing $\mathrm{CaCO}_{3}$ accumulation rates) between 500 and $400 \mathrm{Ka}$, may be related to the so-called Mid-Brunhes Event. According to Jansen et al. (1986), the period between 400 and $300 \mathrm{Ka}$ saw a northward displacement of oceanic fronts that resulted from an intensification of Southern Hemisphere circulation in all ocean basins. However, it appears that the event occurred earlier at Site 846 and that its effect on sea-surface conditions at Site 846 differed significantly from that of the Central Pacific Ocean.

Our record is similar to that described by Schramm (1985) for two cores (V19-29 and RC 10-65) from the eastern equatorial Pacific. Schramm (1985) found that radiolarian faunas indicative of cold water (her "Subpolar Assemblage") had decreased systematically since approximately $500 \mathrm{Ka}$, which she interpreted as a relative decline in the influx of cool, subpolar waters and a warming trend in 
the eastern equatorial Pacific. This warming trend is well represented in our SST data. At the same time, Schramm (1985) found that radiolarian fauna indicative of coastal upwelling and high fertility waters increased steadily from 300 to $50 \mathrm{Ka}$ in Core V19-29 $\left(3^{\circ} 35^{\prime} \mathrm{S}\right.$, $\left.83^{\circ} 56^{\prime} \mathrm{W}\right)$, which is close to Site 846 . Farther west and on the equator, radiolarians in Core $\mathrm{RC} 10-65\left(0^{\circ} 41^{\prime} \mathrm{N}, 108^{\circ} 37^{\prime} \mathrm{W}\right)$ recorded a decrease in the importance of upwelling to approximately $400 \mathrm{Ka}$, followed by a steady increase to $150 \mathrm{Ka}$ and a decrease in upwelling to the present (Schramm, 1985). This pattern of increasing fertility is represented at Site 846 by an increase in TOC accumulation, a decrease in $\mathrm{CaCO}_{3}$ accumulation, and a decrease in dry sediment densities that are attributable to increasing opal contents. Since approximately $120 \mathrm{Ka}(5 \mathrm{mcd})$, increasing sediment densities and increasing $\mathrm{CaCO}_{3}$ accumulation are evident in our record. This can be an indication for decreasing fertility of the surface waters over Site 846 and appears to be consistent with the decrease in faunal upwelling indicators, both at the equator and in the western propagation of the Peru Current (Schramm, 1985).

The faunal and eolian grain-size data set of Core RC11-210 $\left(1^{\circ} 49^{\prime} \mathrm{N}, 140^{\circ} 03^{\prime} \mathrm{W}\right.$; Pisias and Rea, 1988$)$ contains a long record (to $850 \mathrm{Ka}$ ) of the environment in the central equatorial Pacific. Here, a major change in the record character occurred at $300 \mathrm{Ka}$ and is attributed to the mid-Brunhes Event. High amplitude variations before, and a reduction in amplitude after, $300 \mathrm{Ka}$ in sediments of the central equatorial Pacific are a mirror image of the SST and accumulation-rate time series at Site 846 , which display a marked increase in variability toward the top. In addition, the change in record character of both SSTs and accumulation rates occurs approximately 100 k.y. earlier at Site 846 than in the central Equatorial Pacific. The reasons for differences in timing and effect of the event between 400 and $500 \mathrm{Ka}$ between the central and eastern equatorial Pacific are as yet unclear. Analyses of other long records from the equatorial Pacific will help to clarify if these are indeed the same events, if they are propagating from the east to the west, and why their effect is so drastically different nearer to the ocean margin.

\section{Short-term Variability}

Our preliminary analysis of temporal patterns (frequencies and phase relationship with $\delta^{18} \mathrm{O}$ ) in SST and biogenic accumulation rates is limited by the resolution of our records and by the underlying age model: for cross-spectral analyses with the $\delta^{18} \mathrm{O}$ record, SST and accumulation rate records were smoothed with a 24-k.y. Gaussian filter to remove periods $<12 \mathrm{k} . \mathrm{y}$.; these smoothed records were linearly detrended and sampled at 8-k.y. intervals over the period from 0 to 1230 k.y. The records show no coherency in the precession band; however, this could reflect partial attenuation of this frequency by the Gaussian smoothing. Table 2 gives the resulting estimates of coherency and phase between the temperature and accumulation rate records in relation to the $-\delta^{18} \mathrm{O}$ record. Conventionally, a phase of $0^{\circ}$ in the individual frequency bands of eccentricity (1/100 k.y.) and obliquity (1/41 k.y.) corresponds to maxima of the parameter associated with maximal interglacial conditions. In our case, this parameter is $-\delta^{18} \mathrm{O}$ of Mix et al. (this volume). A phase of $\pm 180^{\circ}$ means maximum at glacial extremes of the parameter tested vs. $-\delta^{18} \mathrm{O}$; negative phase leads interglacials, positive phase lags interglacials.

The temperature record is coherent with $-\delta^{18} \mathrm{O}$ over the frequency bands of eccentricity (1/100 k.y.) and of obliquity (1/41 k.y.). However, spectral variance is weak at the frequency of obliquity, as it is in the time series of accumulation rates. In the eccentricity cycle, phase relationships between the SST and isotope records indicate that warmest temperatures lead interglacial events and minimal global ice volume by $7 \pm 5$ k.y. This lead is also seen in the obliquity cycle, where warm SSTs lead $-\delta^{18} \mathrm{O}$ by $5 \pm 2$ k.y.

The time series of organic carbon accumulation is also coherent with $-\delta^{18} \mathrm{O}$ in the eccentricity cycle and its phase corresponds to highest accumulation rates $8 \mathrm{k} . \mathrm{y}$. after glacial maxima of ice volume.
Table 2. Summary of cross-spectral analyses.

\begin{tabular}{|c|c|c|c|c|}
\hline Cycle & $\begin{array}{l}100 \mathrm{k} . \mathrm{y} . \\
\text { Coherency }\end{array}$ & $\begin{array}{l}100 \text { k.y. } \\
\text { Phase }\end{array}$ & $\begin{array}{c}41 \text { k.y. } \\
\text { Coherency }\end{array}$ & $\begin{array}{l}41 \text { k.y. } \\
\text { Phase }\end{array}$ \\
\hline$-\delta^{18} \mathrm{O}$ vs. SST & 0.80 & $-25^{\circ} \pm 18^{\circ}$ & 0.86 & $-43^{\circ} \pm 15^{\circ}$ \\
\hline$-\delta^{18} \mathrm{O}$ vs. $\mathrm{CaCO}_{3}-\mathrm{AR}$ & n.c. & - & 0.91 & $-41^{\circ} \pm 11^{\circ}$ \\
\hline$-\delta^{18} \mathrm{O}$ vs. TOC-AR & 0.81 & $-153^{\circ} \pm 18^{\circ}$ & 0.77 & $11 \mathrm{E} \pm 21^{\circ}$ \\
\hline
\end{tabular}

Notes: Time interval is $0-1.23 \mathrm{Ma} . n=155, t=8 \mathrm{k} . y ., 50$ lags. The $80 \%$ confidence interval for coherency is 0.634 , bandwidth is $0.0033 \mathrm{k} . \mathrm{y}$. $^{-1}$. The figures give phase and coherency estimates relative to $-\delta^{18}$ O. n.c. $=$ not coherent.

Organic carbon accumulation is also coherent with $-\delta^{18} \mathrm{O}$ in the obliquity cycle; in this band, TOC accumulation occurs $3 \mathrm{k} . y$. before maximal glacial conditions. The phase relationship of SST and TOC accumulation to $-\delta^{18} \mathrm{O}$ in the obliquity cycle suggests that maximal TOC accumulation occurs coeval with minimal SST in this cycle. Carbonate accumulation is not coherent with $-\delta^{18} \mathrm{O}$ in the eccentricity cycle and the spectral density of the obliquity cycle is only weak. However, we note similar phase relationships between $\mathrm{CaCO}_{3}$ accumulation and SST over this band, which implies that carbonate accumulation is maximal at warm temperatures in the obliquity cycle.

At this preliminary stage of our analysis, it appears that on glacial/ interglacial time scales, low equatorial SSTs are not linked to increased productivities at Site 846 , because timing of minima in SST and maxima in organic carbon burial have been shifted by approximately 15 k.y. in the coherent $100-k . y$. eccentricity cycle. In a previous investigation, Lyle et al. (1992) used spectral and cross-spectral analyses of insolation, $\delta^{18} \mathrm{O}$ and SST, and accumulation rate time series to elucidate the relationship between climate-forcing mechanisms and timing of maxima in accumulation in relation to upwelling or advection of cold waters in the central equatorial Pacific. An important result of their investigation was that the SSTs there were coherently tied to, but led (5-10 k.y.), global ice volume changes in all primary orbital frequency bands. Our record shows a similar SST coherency with, and lead, over global ice volume in the eccentricity band. The lead of SST over global ice volume is consistent with data from the tropical Atlantic (Imbrie et al., 1989) and appears to be a characteristic and pronounced feature of currents emanating from the eastern boundary currents of the Southern Hemisphere (McIntyre et al., 1989). It reflects an asymmetry in climate change between the two hemispheres and is most likely related to variations in velocity of trade winds and heat advection from high southern latitudes.

The shift in the eccentricity cycle suggests that high productivity is not coeval with minimal temperatures. In our opinion, this argues against periodically intensified upwelling (which should result in low SST) as a dominant factor for organic carbon flux to the sediments. Pedersen (1983), Lyle et al. (1988), and others have proposed that glacial increases in organic carbon burial in the Equatorial Pacific reflect changes in the productivity of surface waters, which are commonly attributed to intensified upwelling during glacial stages (e.g., Müller et al., 1983). This intensified upwelling should be reflected in synchronous negative temperature excursions, which are not evident in our record. Based on the temporal shift in the pronounced eccentricity cycle, Lyle et al. (1992) argued that productivity and biogenic particle flux may be unrelated to upwelling and cold-water advection. Our data agree with this and we propose that the flux of eolian material may be a key factor for determining organic carbon accumulation (but not calcium carbonate accumulation) at Site 846. Observations from productivity patterns in the equatorial Pacific (Chavez et al., 1990), sediment trap data (Ittekkot et al., 1992), and numerous deep-sea cores suggest that organic carbon production, its transfer through the water column, and its accumulation in the sediment are intimately tied to the flux of eolian aluminosilicate material. We propose that increased burial rates of organic carbon during glacials may be in part an effect of increased eolian influx into the area at glacial maxima (as proposed for nearby Core V19-29 in the late Quaternary by Boyle, 1983). Increased eolian influx may have pro- 
vided micronutrients for organic carbon production, ballast for a rapid transfer through the water column, and a seal against remineralization in the sediment (Davidson et al., in press).

In the shorter 41-k.y. cycle of obliquity, there is indication of a direct link between TOC accumulation and colder, possibly nutrientrich surface waters. The signal of the obliquity cycle is weak in all time series, however, and clearly is not as important as the process varying at the periodicity of 100 k.y.

\section{CONCLUSIONS}

Our reconstruction of biogenic accumulation rates and SSTs have shown that sea-surface conditions at the transition from the Peru Current to the SEC of the eastern equatorial Pacific have fluctuated strongly over the past $1.3 \mathrm{~m} . \mathrm{y}$. A long trend of gradual decrease in SSTs from $24^{\circ} \mathrm{C}$ at $1.3 \mathrm{Ma}$ ends between 500 and $400 \mathrm{Ka}$, when lowest values of $19^{\circ} \mathrm{C}$ were reached. Since this time, the temperature data indicate a warming trend to the Holocene modulated by highamplitude variation $\left(19^{\circ}-27^{\circ} \mathrm{C}\right)$. The inversion of the trend between 400 and $500 \mathrm{Ka}$ coincides with maximal accumulation rates of carbonate, which since then have decreased. In contrast, organic carbon accumulation has since then increased in variability and in absolute values. On shorter time scales, conditions at the sea surface reflect strong links to glacial/interglacial cycles over much of the Pleistocene era. In general, SST is high and productivity is low during interglacials. However, differences in their timing with respect to the $\delta^{18} \mathrm{O}$ record of benthic foraminifers (Mix et al., this volume) suggest that temperature variations on glacial/interglacial time scales are not immediately linked to productivity and/or particle flux.

\section{ACKNOWLEDGMENTS}

We thank all participants of Leg 138 for a stimulating cruise, N. Pisias, F. Prahl, S. Stewart, and K. Winn for thorough reviews, and the Deutsche Forschungsgemeinschaft (DFG EM-37) for funding our research.

\section{REFERENCES*}

Boyle, E.A., 1983. Chemical accumulation variations under the Peru Current during the past 130,000 years. J. Geophys. Res., 88:7667-7680.

Brassell, S.C., Eglinton, G., Marlowe, I.T., Pflaumann, U., and Sarnthein, M., 1986. Molecular stratigraphy: a new tool for climatic assessment. Nature, 320:129-133.

Bryden, H.L., and Brady, E.C., 1985. Diagnostic model of the three-dimensional circulation in the upper Equatorial Pacific Ocean. J. Phys. Oceanogr., 15:1255-1273.

Chavez, F., Buck, K.R., and Barber, R.T., 1990. Phytoplankton taxa in relation to primary productivity in the equatorial Pacific. Deep-Sea Res. Part A, 37:1733-1752.

Chavez, F.P., and Barber, R.T., 1987. An estimate of new production in the equatorial Pacific. Deep-Sea Res. Part A, 34:1229-1243.

Dandonneau, Y., and Eldin, G., 1987. Southward extent of chlorophyllenriched waters from the Peruvian and equatorial upwellings between Tahiti and Panama. Mar. Ecol, Prog. Ser., 38:283-294.

Davidson, E., Ågren, G., Daniel, O., Emeis, K.-C., Largeau, C., Lee, C., Mopper, K., Oades, J., Reeburgh, B., Schimel, D., et al., in press. What are the physical, chemical, and biological processes that control the formation and degradation of nonliving organic matter? In Zepp, R., and Sonntag, C. (Eds.), The Role of Nonliving Organic Matter in the Earth's Carbon Cycle: Chichester (Wiley).

Eglinton, G., Bradshaw, S.A., Rosell, A., Sarnthein, M., Pflaumann, U., and Tiedemann, R., 1992. Molecular record of sea surface temperature changes on 100-year time scales for glacial terminations I, II, and IV. Nature, 356:423-426.

Halpern, D., Ashby, H., Finch, C., and Robles, J., 1990. TOGA CD-ROM description. JPL Publ., 90-43.

Imbrie, J., McIntyre, A., and Mix, A., 1989. Oceanic response to orbital forcing in the late Quaternary: observational and experimental strategies. In Berger, A., Schneider, S., and Duplessy, J.C. (Eds.), Climate and Geo-Sciences: Dordrecht (Kluwer Academic), 121-164.

Ittekkot, V., Haake, B., Bartsch, M., Nair, R.R., and Ramaswami, V., 1992. Organic carbon removal in the sea: the continental connection. In Summerhayes, C.P., Prell, W.L., and Emeis, K.-C. (Eds.), Upwelling System: Evolution Since the Early Miocene. Geol. Soc. Spec. Publ. London, $167-176$.

Jansen, J.H.F., Kuijpers, A., and Troelstra, S.R., 1986. Amid-Brunhes climatic event: long-term changes in global atmospheric and ocean circulation. Science, 232:619-622.

Lyle, M., 1992. Composition maps of surface sediments of the eastern tropical Pacific Ocean. In Mayer, L., Pisias, N., Janecek, T., et al., Proc. ODP, Init. Repts., 138 (Pt. 1): College Station, TX (Ocean Drilling Program), 101-115.

Lyle, M., Murray, D.W., Finney, B.P., Dymond, J., Robbins, J.M., and Brooksforce, K., 1988. The record of late Pleistocene biogenic sedimentation in the eastern tropical Pacific Ocean. Paleoceanography, 3:39-59.

Lyle, M.W., Prahl, F.G., and Sparrow, M.A., 1992. Upwelling and productivity changes inferred from a temperature record in the central equatorial Pacific. Nature, 355:812-815.

Marlowe, I.T., Brassell, S.C., Eglinton, G., and Green, J.C., 1990. Long-chain alkenones and alkyl alkenoates and the fossil coccolith record of marine sediments. Chem. Geol., 88:349-375.

Mayer, L., Pisias, N., Janecek, T., et al., 1992. Proc. ODP, Init. Repts., 138 (Pts. 1 and 2): College Station, TX (Ocean Drilling Program).

McIntyre, A., Ruddiman, W.F., Karlin, K., and Mix, A.C., 1989. Surface water response of the equatorial Atlantic Ocean to orbital forcing. Paleoceanography, 4:19-55.

Müller, P.J., Erlenkeuser, H., and von Grafenstein, R., 1983. Glacial-interglacial cycles in oceanic productivity inferred from organic carbon contents in eastern North Atlantic sediment cores. In Suess, E., and Thiede, J. (Eds.), Coastal Upwelling: Its Sediment Record (Pt. B.): New York (Plenum), 365-398.

Pedersen, T.F., 1983. Increased productivity in the eastern equatorial Pacific during the last glacial maximum (19,000 to $14,000 \mathrm{yr}$ B.P.). Geology, 11:16-19.

Pisias, N., and Rea, D.K., 1988. Late Pleistocene paleoclimatology of the central equatorial Pacific: sea surface response to the southeast trade winds. Paleoceanography, 3:21-37.

Prahl, F.G., Muehlhausen, L.A., and Zahnle, D.L., 1988. Further evaluation of long-chain alkenones as indicators of paleoceanographic conditions. Geochim. Cosmochim. Acta, 52:2303-2310.

Schramm, C.T., 1985. Implications of radiolarian assemblages for the Late Quaternary paleoceanography of the eastern equatorial Pacific. Quat. Res. (N.Y.), 24:204-218.

Wooster, W.S., 1969. Equatorial front between Peru and Galapagos. Deep-Sea Res. Part A, 16:407-419.

Wyrtki, K., 1981. An estimate of equatorial upwelling in the Pacific. J. Phys. Oceanogr., 11:1205-1214.

Abbreviations for names of organizations and publication titles in ODP reference lists
follow the style given in Chemical Abstracts Service Source Index (published by follow the style given in Chem
American Chemical Society).

Date of initial receipt: 1 February 1993

Date of acceptance: 31 December 1993

Ms 138SR-131 